\title{
PRIMES IN ARITHMETIC PROGRESSIONS
}

\author{
OLIVIER RAMARÉ AND ROBERT RUMELY
}

\begin{abstract}
Strengthening work of Rosser, Schoenfeld, and McCurley, we establish explicit Chebyshev-type estimates in the prime number theorem for
\end{abstract} arithmetic progressions, for all moduli $k \leq 72$ and other small moduli.

\section{INTRODUCTION}

In many applications it is useful to have explicit error bounds in the prime number theorem for arithmetic progressions. Furthermore, in numerical work, such estimates for small moduli are often the most critical. Let us recall the usual notation, where $x \geq 0$ is real:

$$
\begin{aligned}
\theta(x ; k, l) & =\sum_{\substack{p \equiv l(k) \\
p \leq x}} \ln (p), \quad \text { where } p \text { denotes a prime number; } \\
\psi(x ; k, l) & =\sum_{\substack{n \equiv l(k) \\
n \leq x}} \Lambda(n), \quad \text { where } \Lambda(n) \text { is Von Mangoldt's function. }
\end{aligned}
$$

Here we obtain estimates of the type

$$
(1-\epsilon) \frac{x}{\varphi(k)} \leq \theta(x ; k, l) \leq \psi(x ; k, l) \leq(1+\epsilon) \frac{x}{\varphi(k)}
$$

for all moduli $k \leq 72$, all composite $k \leq 112$, and 48 other moduli; $\varphi(k)$ denotes Euler's function.

To obtain such estimates for the progression with modulus 1, Rosser and Schoenfeld $([8,9,11])$ developed an analytic method which combines a numerical verification of the Riemann Hypothesis to a given height together with an explicit asymptotic zero-free region. McCurley $([3,4,5,6])$ adapted this method to progressions with modulus $k>1$. However, except for $k=3$, he got poor numerical results because of the paucity of numerical work on the Generalized Riemann Hypothesis. Recently, the second author [10] did such computations, and we get reasonably good results for all moduli accessible from his list. In fact, the results are better than one would expect, owing to a smoothing process implicit in the method, explained in $\S 4.4$.

Received by the editor February 26, 1993 and, in revised form, January 24, 1994, June 27, 1994, and January 10, 1995.

1991 Mathematics Subject Classification. Primary 11N13, 11N56, 11M26; Secondary 11Y35, $11 \mathrm{Y} 40,11-04$. 
Our main theoretical result is Theorem 4.3.2, which provides an easy way to get applications like Theorem 1 below. In Theorem 3.6.2 we also establish a zerofree region for Dirichlet $L$-functions, valid for heights $|t| \geq 1000$, which improves somewhat on the region found by McCurley [4].

Finally, we mention that this paper is an important step in a forthcoming paper by the first author, in which it is proved that every even integer can be written as a sum of at most 6 primes.

Our main numerical result is the following:

Theorem 1. For any triple $\left(k, \varepsilon, x_{0}\right)$ given by Table 1 (see $\left.\S 5\right)$, and any $l$ prime to $k$, we have

$$
\begin{aligned}
& \max _{1 \leq y \leq x}\left|\theta(y ; k, l)-\frac{y}{\varphi(k)}\right| \leq \varepsilon \frac{x}{\varphi(k)} \quad \text { for } x \geq x_{0}, \\
& \max _{1 \leq y \leq x}\left|\psi(y ; k, l)-\frac{y}{\varphi(k)}\right| \leq \varepsilon \frac{x}{\varphi(k)} \quad \text { for } x \geq x_{0} .
\end{aligned}
$$

For example, if $k=1$,

$$
\begin{gathered}
\varepsilon=0.000213 \text { for } x_{0}=10^{10}, \quad \varepsilon=0.000015 \text { for } x_{0}=10^{13}, \\
\varepsilon=0.000001 \text { for } x_{0}=10^{30} .
\end{gathered}
$$

If $k \leq 13$, one can take

$$
\begin{aligned}
& \varepsilon=0.004560 \text { for } x_{0}=10^{10}, \quad \varepsilon=0.002657 \text { for } x_{0}=10^{13} \\
& \varepsilon=0.002478 \text { for } x_{0}=10^{30}, \quad \varepsilon=0.002020 \text { for } x_{0}=10^{100}
\end{aligned}
$$

If $k \leq 72$, one can take

$$
\begin{aligned}
& \varepsilon=0.023269 \text { for } x_{0}=10^{10}, \quad \varepsilon=0.011310 \text { for } x_{0}=10^{13} \\
& \varepsilon=0.010484 \text { for } x_{0}=10^{30}, \quad \varepsilon=0.008672 \text { for } x_{0}=10^{100} .
\end{aligned}
$$

We have supplemented these analytic results with a tabulation up to $10^{10}$, obtaining surprisingly uniform bounds:

Theorem 2. For all moduli $k$ in Table 1, and all $l$ prime to $k$, uniformly for $1 \leq x \leq 10^{10}$,

$$
\begin{aligned}
& \max _{1 \leq y \leq x}\left|\theta(y ; k, l)-\frac{y}{\varphi(k)}\right| \leq 2.072 \sqrt{x} \\
& \max _{1 \leq y \leq x}\left|\psi(y ; k, l)-\frac{y}{\varphi(k)}\right| \leq 1.745 \sqrt{x} .
\end{aligned}
$$

In particular, for a bound of the type in Theorem 1 when $x_{0} \leq x \leq 10^{10}$, one can take

$$
\varepsilon=2.072 \varphi(k) / \sqrt{x}_{0} .
$$

Sharper bounds for individual moduli are given in Table 2 (see §5). Especially, for $k=5$ or $k \geq 7$, the constant 1.745 in the error bound for $\psi(x ; k, l)$ can be replaced by 1.000 , and for $k=1$ it can be replaced by $\sqrt{2}$. 


\section{Numerical RESUlts about the GRH}

Throughout the paper, $p$ always stands for a prime and the gcd of $k$ and $l$ is written $(k, l)$.

The letter $\rho$ always denotes a nontrivial zero of a Dirichlet $L$-function, i.e., a zero with $0<\Re \rho<1$. We will always write $\rho=\beta+i \gamma$.

Given a Dirichlet $L$-function $L(s, \chi)$, the set of its zeros with $0<\beta<1$ will be denoted by $\mathcal{Z}(\chi)$. Thus, if $\chi^{\prime}$ is induced by $\chi$, then $\mathcal{Z}\left(\chi^{\prime}\right)=\mathcal{Z}(\chi)$.

Given a nonnegative real number $H$, we will say that $L(s, \chi)$ satisfies $\operatorname{GRH}(H)$, the Generalized Riemann Hypothesis to height $H$, if all its nontrivial zeros with $|\gamma| \leq H$ verify $\beta=\frac{1}{2}$.

It is possible to prove or disprove $\operatorname{GRH}(H)$ for any given $H$ and $L(s, \chi)$ with only a finite amount of computation. Rumely [10] did such computations, obtaining:

\section{Theorem 2.1.1.}

- Every L-function associated with a Dirichlet character of conductor $k \leq 13$ satisfies GRH(10000).

- Every L-function associated with a Dirichlet character of conductor in the sets

$$
\begin{aligned}
& \{k \leq 72\}, \\
& \{k \leq 112, k \text { not prime }\} \\
& \{116,117,120,121,124,125,128,132,140,143,144, \\
& \quad 156,163,169,180,216,243,256,360,420,432\}
\end{aligned}
$$

satisfies $\operatorname{GRH}(2500)$.

Sharper results for individual moduli, together with bounds for the sums

$$
\sum_{\substack{|\operatorname{Im}(\rho)| \leq H \\ \rho \in \mathcal{Z}(\bar{\chi})}} \frac{1}{|\rho|}
$$

may be found in Tables 1 and 5 of [10]; these results were used in computing Table 1 below. The following facts about the Riemann zeta function were also used:

$$
\zeta(s) \text { satisfies GRH(545439823.215) }
$$

(van de Lune, te Riele, and Winter [2]);

$$
\text { for } H=12030, \sum_{|\operatorname{Im}(\rho)| \leq H} \frac{1}{|\rho|} \leq 9.056
$$

(R. S. Lehman, cf. Rosser and Schoenfeld [9]).

\section{ZERO-FREE REGIONS FOR $L$-FUNCTIONS}

This part follows McCurley [4] and some evaluations are taken directly from McCurley's paper without being re-established. We employ a device due to Stechkin $[12]$ to widen the zero-free region.

Let $s=\sigma+i t$ and $s_{1}=\sigma_{1}+i t$ be complex numbers with $|t| \geq 1000$ satisfying

$$
\left\{\begin{array}{l}
1<\sigma \leq \frac{1+\sqrt{2}}{2} \leq \sigma_{1} \leq \frac{1+\sqrt{5}}{2} \\
\sqrt{\sigma(\sigma-1)}+\sqrt{\sigma_{1}\left(\sigma_{1}-1\right)}=1
\end{array}\right.
$$


and put $\kappa(\sigma)=\frac{1+\frac{4}{5} \sqrt{\sigma-1}}{\sqrt{5}}$. Note that $1 / \sqrt{5} \leq \kappa(\sigma) \leq 0.61004$, and that $\sigma \geq \kappa(\sigma) \sigma_{1}$.

These assumptions on $\sigma$ and $\sigma_{1}$ will hold throughout the paper.

We define the auxiliary function

$$
f(t, \chi)=\Re\left(\sum_{n \geq 1} \Lambda(n)\left(\frac{1}{n^{\sigma}}-\frac{\kappa(\sigma)}{n^{\sigma_{1}}}\right) \frac{\chi(n)}{n^{i t}}\right)
$$

and introduce constants

$$
\left\{\begin{array} { l } 
{ \varepsilon _ { 1 } = 0 . 0 0 6 7 , } \\
{ \varepsilon _ { 2 } = 1 0 ^ { - 6 } , }
\end{array} \quad \left\{\begin{array}{l}
a_{0}=11.1859355312082048 \\
a_{1}=19.073344004352 \\
a_{2}=11.67618784 \\
a_{3}=4.7568 \\
a_{4}=1 \\
A=a_{1}+a_{2}+a_{3}+a_{4}=36.506331844352
\end{array}\right.\right.
$$

\subsection{A device of Stechkin.}

Lemma 3.1.1 (Stechkin). Let $z$ be a complex number with $0 \leq \Re z \leq 1$. Put

$$
F(s, z)=\Re\left\{\frac{1}{s-z}+\frac{1}{s-(1-\bar{z})}\right\} .
$$

Then

$$
F(s, z) \geq \kappa(\sigma) F\left(s_{1}, z\right) .
$$

Moreover, if $\Im z=\Im s$ and $\frac{1}{2} \leq \Re z \leq 1$, and if $S(\sigma)=-\frac{1}{\sigma}+\kappa(\sigma)\left(\frac{1}{\sigma_{1}}+\frac{1}{\sigma_{1}-1}\right)$, then

$$
-\Re\left(\frac{1}{s-(1-\bar{z})}\right)+\kappa(\sigma) F\left(s_{1}, z\right) \leq S(\sigma) .
$$

Proof. The first inequality is implicit in Stechkin [12]: see the top line on p.132 of the English translation. For the second inequality, note that when $\Im z=\Im s=t$, then, writing $z=\beta+i t$, the left side of the inequality becomes

$$
-\frac{1}{\sigma-1+\beta}+\kappa(\sigma)\left(\frac{1}{\sigma_{1}-\beta}+\frac{1}{\sigma_{1}-1+\beta}\right) .
$$

For $\frac{1}{2} \leq \beta \leq 1$, both terms are increasing with $\beta$. Taking $\beta=1$ we obtain the result.

As will be seen later, we will only need to consider $\sigma$ in the interval $[1,1.062]$. Although we will not use this, it can be shown that on $[1,1.062]$

$$
S(\sigma)=2 \sqrt{\Delta}+\frac{86}{25} \Delta+\mathcal{O}\left(\Delta^{3 / 2}\right),
$$

where $\Delta=\sigma-1$ and the implied constant can be taken as 18. Furthermore, throughout the interval,

$$
2 \sqrt{\Delta}+3 \Delta \leq S(\sigma) \leq 2 \sqrt{\Delta}+5 \Delta .
$$




\subsection{Handling the $\Gamma$-factor.}

Lemma 3.2.1. If $0 \leq a \leq 2, \varepsilon_{1}=0.0067$ and $\varepsilon_{2}=10^{-6}$, then for $|t| \geq 1000$

$$
\frac{1}{2} \Re\left\{\frac{\Gamma^{\prime}}{\Gamma}\left(\frac{s+a}{2}\right)-\kappa(\sigma) \frac{\Gamma^{\prime}}{\Gamma}\left(\frac{s_{1}+a}{2}\right)\right\} \leq \frac{1-\kappa(\sigma)}{2}\left(\ln \left(\frac{|t|}{2}\right)+\varepsilon_{1}-10 \varepsilon_{2}\right) .
$$

Proof. We follow Lemma 2 of McCurley ([4]). Since $\Re\left(\frac{\Gamma^{\prime}}{\Gamma}(z)\right)$ is invariant under $z \rightarrow \bar{z}$, we have for $z=x+i y$, with $x>0$ :

$$
\Re \frac{\Gamma^{\prime}}{\Gamma}\left(\frac{z+a}{2}\right)=\ln \left(\frac{|y|}{2}\right)+\frac{1}{2} \ln \left|1+\left(\frac{x+a}{y}\right)^{2}\right|-\frac{x+a}{(x+a)^{2}+y^{2}}+\Theta(y)
$$

where $|\Theta(y)| \leq \frac{1}{|y|} \cdot\left[\frac{\pi}{2}-\arctan \left(\frac{x+a}{|y|}\right)\right] \leq\left|\frac{\pi}{2 y}\right|$. We apply this, taking $z=s, s_{1}$. Using $\sigma \geq \kappa(\sigma) \sigma_{1}$, we find

$$
\left\{\begin{array}{l}
-\frac{\sigma+a}{(\sigma+a)^{2}+t^{2}}+\kappa(\sigma) \frac{\sigma_{1}+a}{\left(\sigma_{1}+a\right)^{2}+t^{2}} \leq 0 \\
\frac{1}{4} \ln \left|1+\left(\frac{\sigma+a}{t}\right)^{2}\right| \leq \frac{2.58}{t^{2}} \\
\frac{1}{2}(1+\kappa(\sigma)) \Theta(t) \leq \frac{1.26453}{|t|} .
\end{array}\right.
$$

These facts, together with $(1-\kappa(\sigma)) / 2 \geq 0.19498$, yield the result.

3.3. Approximating $f(t, \chi)$. Here the character which is the argument of $f(t, \cdot)$ is assumed to be primitive.

Lemma 3.3.1. If $\chi_{0}$ is the trivial character and

$$
E(\sigma)=1.08699+1.40018 \sqrt{\sigma-1}+1.86576(\sigma-1)+2.32244(\sigma-1)^{3 / 2},
$$

then for $1<\sigma \leq 1.062$,

$$
f\left(0, \chi_{0}\right) \leq \frac{1}{\sigma-1}-E(\sigma)
$$

Proof. We follow McCurley's Lemma 3 ([4]). We have for any complex number $z$

$$
-\frac{\zeta^{\prime}}{\zeta}(z)=\frac{1}{z-1}-\frac{\ln (\pi)}{2}+\frac{\Gamma^{\prime}}{2 \Gamma}\left(\frac{z+2}{2}\right)-\sum_{\rho \in \mathcal{Z}\left(\chi_{0}\right)} \frac{1}{z-\rho},
$$

where $\sum_{\rho}$ is to be understood as $\lim _{T \rightarrow \infty} \sum_{\rho,|\gamma| \leq T}$. We use (3.3.1) for $z=\sigma$. Since for any zero of $\zeta$, we have $|\gamma| \geq 10 \geq \sigma-\beta$, we see that

$$
\frac{\sigma-\beta}{(\sigma-\beta)^{2}+\gamma^{2}} \geq \frac{1-\beta}{(1-\beta)^{2}+\gamma^{2}} .
$$


It follows that

$$
\begin{aligned}
f\left(0, \chi_{0}\right) & =-\frac{\zeta^{\prime}}{\zeta}(\sigma)+\kappa(\sigma) \frac{\zeta^{\prime}}{\zeta}\left(\sigma_{1}\right) \\
& \leq \frac{1}{\sigma-1}-\frac{\ln (\pi)}{2}+\frac{\Gamma^{\prime}}{2 \Gamma}\left(\frac{\sigma+2}{2}\right)-\Re\left(\sum_{\rho \in \mathcal{Z}\left(\chi_{0}\right)} \frac{1}{1-\rho}\right)+\kappa(\sigma) \frac{\zeta^{\prime}}{\zeta}\left(\sigma_{1}\right) .
\end{aligned}
$$

We recall McCurley's estimate (taken from the proof of Lemma 3 of [4])

$$
\frac{\Gamma^{\prime}}{2 \Gamma}\left(\frac{\sigma+2}{2}\right) \leq 1-\frac{C_{0}}{2}-\ln (2)+\left(\frac{\pi^{2}}{8}-1\right)(\sigma-1),
$$

where $C_{0} \cong 0.5772156649$ denotes Euler's constant. Further, by (Landau $[1, \S 76$, pp. 316-317]),

$$
\sum_{\rho} \frac{1}{1-\rho}=\sum_{\rho} \frac{1}{\rho}=-\frac{1}{2} \ln (\pi)-\ln (2)+1+\frac{C_{0}}{2} .
$$

Thus, we can take

$$
E(\sigma)=C_{0}-\left(\frac{\pi^{2}}{8}-1\right)(\sigma-1)+\kappa(\sigma)\left(-\frac{\zeta^{\prime}}{\zeta}\left(\sigma_{1}\right)\right) .
$$

We now estimate $-\left(\zeta^{\prime} / \zeta\right)\left(\sigma_{1}\right)$, which is a decreasing, nonnegative function of $\sigma_{1}$. As $\sigma \rightarrow 1^{+}, \sigma_{1}$ approaches $(1+\sqrt{5}) / 2$ from below. Writing $\Delta=\sigma-1$, we have

$$
\sigma_{1}=\frac{1+\sqrt{5} \sqrt{1-\left(\frac{8}{5}\right) \Delta^{1 / 2}+\left(\frac{4}{5}\right) \Delta-\left(\frac{4}{5}\right) \Delta^{3 / 2}+\left(\left(\frac{4}{5}\right) \Delta^{2}+\left(\frac{1}{5}\right) \Delta^{5 / 2}\right)-\ldots}}{2} .
$$

For $0 \leq \Delta \leq 0.062$ the series inside the radical is alternating, with decreasing terms. Thus

$$
\sigma_{1} \leq \frac{1+\sqrt{5} \sqrt{1-\left(\frac{8}{5}\right) \Delta^{1 / 2}+\left(\frac{4}{5}\right) \Delta}}{2} .
$$

Upon expanding and using $\Delta \leq 0.062$, we obtain

$$
\begin{aligned}
\sigma_{1} & \leq \frac{1+\sqrt{5}\left(1-\frac{1}{2}\left(\frac{8}{5} \Delta^{1 / 2}-\frac{4}{5} \Delta\right)-\frac{1}{8}\left(\frac{8}{5} \Delta^{1 / 2}-\frac{4}{5} \Delta\right)^{2}\right)}{2} \\
& \leq \frac{1+\sqrt{5}\left(1-\left(\frac{4}{5}\right) \Delta^{1 / 2}+\left(\frac{4}{25}\right) \Delta\right)}{2} .
\end{aligned}
$$

It follows that

$$
(1+\sqrt{5}) / 2-\sigma_{1} \geq\left(\frac{2}{\sqrt{5}}\right) \Delta^{1 / 2}-\left(\frac{2}{5 \sqrt{5}}\right) \Delta .
$$

When $-\left(\zeta^{\prime} / \zeta\right)\left(\sigma_{1}\right)$ is developed as a Taylor series about $(1+\sqrt{5}) / 2$, its coefficients alternate in sign. The first four coefficients satisfy

$$
B_{0} \geq 1.13991, \quad-B_{1} \geq 2.48089, \quad B_{2} \geq 4.20397, \quad-B_{3} \geq 6.84664 ;
$$


these values were obtained by expressing the coefficients in terms of the derivatives of the zeta function, which were computed using Euler-Maclaurin summation. Thus,

$$
\begin{aligned}
-\left(\zeta^{\prime} / \zeta\right)\left(\sigma_{1}\right) \geq 1.13991 & +2.48089\left(\frac{2 \sqrt{\Delta}}{\sqrt{5}}-\frac{2}{5 \sqrt{5}} \Delta\right) \\
& +4.20397\left(\frac{2 \sqrt{\Delta}}{\sqrt{5}}-\frac{2}{5 \sqrt{5}} \Delta\right)^{2}+6.84664(0.84988 \sqrt{\Delta})^{3} \\
\geq 1.13991 & +2.21897 \sqrt{\Delta}+2.91938 \Delta+2.85764 \Delta^{3 / 2}
\end{aligned}
$$

(Here we have again used that $0 \leq \Delta \leq 0.062$.) Inserting this in (3.3.4) yields

$$
E(\sigma) \geq 1.08699+1.40018 \Delta^{1 / 2}+1.86576 \Delta+2.32244 \Delta^{3 / 2}
$$

and for the purposes of the lemma we can replace $E(\sigma)$ by its bound.

Lemma 3.3.2. If $\chi$ is a character of conductor $k$, then for $|t| \geq 1000$ and $\varepsilon_{1}=$ 0.0067 ,

$$
\begin{aligned}
f(t, \chi) \leq & \frac{1-\kappa(\sigma)}{2}\left(\ln \left(\frac{k|t|}{2 \pi}\right)+\varepsilon_{1}\right) \\
& -\Sigma^{\prime} \Re\left\{\frac{1}{s-\rho}+\frac{1}{s-(1-\bar{\rho})}-\frac{\kappa(\sigma)}{s_{1}-\rho}-\frac{\kappa(\sigma)}{s_{1}-(1-\bar{\rho})}\right\},
\end{aligned}
$$

where $\Sigma^{\prime}$ denotes a sum over the zeros of $L(s, \chi)$ with $\beta \geq 1 / 2$ and where the zeros with $\beta=1 / 2$ have a weight $\frac{1}{2}$.

Proof. We consider separately the cases $k=1$ and $k>1$.

First suppose $k=1$. Using (3.3.1) with $z=s, s_{1}$, we get

$$
\begin{gathered}
f\left(t, \chi_{0}\right)=\Re\left\{\frac{1}{s-1}-\frac{\kappa(\sigma)}{s_{1}-1}\right\}+\frac{1}{2} \Re\left\{\frac{\Gamma^{\prime}}{\Gamma}\left(\frac{s+2}{2}\right)-\kappa(\sigma) \frac{\Gamma^{\prime}}{\Gamma}\left(\frac{s_{1}+2}{2}\right)\right\} \\
-\frac{1-\kappa(\sigma)}{2} \ln (\pi)-\frac{1}{2} \sum_{\rho \in \mathcal{Z}\left(\chi_{0}\right)}\left(F(s, \rho)-\kappa(\sigma) F\left(s_{1}, \rho\right)\right) .
\end{gathered}
$$

By the functional equation of $\zeta(s)$, the last sum can be rewritten

$$
\Sigma^{\prime} \Re\left(\frac{1}{s-\rho}+\frac{1}{s-(1-\bar{\rho})}-\frac{\kappa(\sigma)}{s_{1}-\rho}-\frac{\kappa(\sigma)}{s_{1}-(1-\bar{\rho})}\right),
$$

where the notation is as in the statement of the lemma.

Lemma 3.2.1, together with

$$
\Re\left\{\frac{1}{s-1}-\frac{\kappa(\sigma)}{s_{1}-1}\right\} \leq \frac{\sigma-1}{t^{2}} \leq \varepsilon_{2}
$$

and $(1-\kappa(\sigma)) / 2 \geq 0.19498$, yields the result.

Now suppose $k>1$. The proof is similar to the previous one, apart from the absence of the pole at $s=1$. Following McCurley's Lemma 5 ([4]), we have

$$
\begin{aligned}
f(t, \chi)= & \frac{1-\kappa(\sigma)}{2} \ln \left(\frac{k}{\pi}\right)+\frac{1}{2} \Re\left\{\frac{\Gamma^{\prime}}{\Gamma}\left(\frac{s+a}{2}\right)-\kappa(\sigma) \frac{\Gamma^{\prime}}{\Gamma}\left(\frac{s_{1}+a}{2}\right)\right\} \\
& -\Sigma^{\prime}\left(F(s, \rho)-\kappa(\sigma) F\left(s_{1}, \rho\right)\right)
\end{aligned}
$$

with $a=(1-\chi(-1)) / 2$, and Lemma 3.2.1 gives the required estimate.

Using Lemmas 3.1.1 and 3.3.2, we get 
Lemma 3.3.3. If $\chi$ is a character of conductor $k$, then for $|t| \geq 1000$ and $\varepsilon_{1}=$ 0.0067

$$
f(t, \chi) \leq \frac{1-\kappa(\sigma)}{2}\left(\ln \left(\frac{k|t|}{2 \pi}\right)+\varepsilon_{1}\right)
$$

and if $t=\gamma$ is the ordinate of a zero $\rho=\beta+i \gamma$ with $\beta>\frac{1}{2}$, then

$$
f(t, \chi) \leq \frac{1-\kappa(\sigma)}{2}\left(\ln \left(\frac{k|t|}{2 \pi}\right)+\varepsilon_{1}\right)-\frac{1}{\sigma-\beta}+S(\sigma) .
$$

3.4. The default to primitivity. Let $\chi$ be a primitive character of conductor $k$ and let $\chi_{m}$ be the primitive character associated with $\chi^{m}$, of conductor $k_{m}$. For a prime $p$ dividing $k$ we put

$$
\left\{\begin{array}{l}
c_{p}(\sigma)=\frac{1}{p^{\sigma}-1}-\frac{\kappa(\sigma)}{p^{\sigma_{1}}-1}, \\
D_{p}(k, \sigma, \chi)=a_{0} c_{p}(\sigma)+\sum_{\substack{1 \leq m \leq 4 \\
p \nmid k_{m}}} a_{m}\left(\frac{1-\kappa(\sigma)}{2} v_{p}\left(\frac{k}{k_{m}}\right)-c_{p}(\sigma)\right),
\end{array}\right.
$$

where $v_{p}(x)$ is the $p$-adic valuation of $x$ with $v_{p}(p)=1$. We seek a lower bound for

$$
D(k, \sigma, \chi)=\sum_{p \mid k} \ln (p) D_{p}(k, \sigma, \chi) .
$$

This bound will be $D^{*}(k, \sigma)$, defined below: put

$$
\left\{\begin{array}{l}
D_{2}^{*}(\sigma)=5.99 c_{2}(\sigma), \\
D_{3}^{*}(\sigma)=5.467 c_{3}(\sigma), \\
D_{p}^{*}(\sigma)=a_{0} c_{p}(\sigma), \text { for } p \geq 5
\end{array}\right.
$$

and

$$
D^{*}(k, \sigma)=\sum_{p \mid k} \ln (p) D_{p}^{*}(\sigma) .
$$

Lemma 3.4.1. The quantity $c_{p}(\sigma)$ is positive, and decreases as $p$ increases or as $\sigma$ increases.

Proof. Easy.

Lemma 3.4.2. We have

$$
\left\{\begin{array}{l}
\frac{1-\kappa(\sigma)}{c_{2}(\sigma)}-1 \geq-0.298 \\
\frac{1-\kappa(\sigma)}{2 c_{3}(\sigma)}-1 \geq-0.328 \\
\frac{1-\kappa(\sigma)}{2}-c_{5}(\sigma) \geq 0
\end{array}\right.
$$

Proof. If $\sigma^{-} \leq \sigma \leq \sigma^{+}$, then

$$
\frac{1-\kappa\left(\sigma^{+}\right)}{2}-c_{5}\left(\sigma^{-}\right) \leq \frac{1-\kappa(\sigma)}{2}-c_{5}(\sigma) \leq \frac{1-\kappa\left(\sigma^{-}\right)}{2}-c_{5}\left(\sigma^{+}\right) .
$$

Cutting the interval $[1,(1+\sqrt{2}) / 2]$ into 10000 parts yields the third inequality in the lemma. The two other inequalities may be obtained similarly. 
Lemma 3.4.3. For each prime $p \mid k$, we have $D_{p}(k, \sigma, \chi) \geq D_{p}^{*}(\sigma)$.

Proof. If $p \geq 5$, then $c_{5}(\sigma) \geq c_{p}(\sigma)$, so by Lemma 3.4.2 and the fact that $k_{1}=k$, we see that for each prime dividing $k$,

$$
\begin{aligned}
D_{p}(\kappa, \sigma, \chi) & =a_{0} c_{p}(\sigma)+\sum_{\substack{m=2 \\
p \nmid k_{m}}}^{4} a_{m}\left(\frac{1-\kappa(\sigma)}{2} v_{p}\left(\frac{k}{k_{m}}\right)-c_{p}(\sigma)\right) \\
& \geq a_{0} c_{p}(\sigma)=D_{p}^{*}(\sigma) .
\end{aligned}
$$

The proof is analogous for $p=2$ and $p=3$. Note that when $p=2$, if $p \nmid k_{m}$, then $v_{p}\left(\frac{k}{k_{m}}\right) \geq 2$. The constant in $D_{2}^{*}(\sigma)$ is $a_{0}-0.298\left(a_{2}+a_{3}+a_{4}\right)$, and the one in $D_{3}^{*}(\sigma)$ is $a_{0}-0.328\left(a_{2}+a_{3}+a_{4}\right)$.

Combining Lemmas 3.4.1 and 3.4.2 gives the desired result:

Corollary 3.4.4. For $1 \leq \sigma \leq(1+\sqrt{2}) / 2$, we have $D(k, \sigma, \chi) \geq D^{*}(k, \sigma)$. Furthermore, on this interval $D^{*}(k, \sigma)$ is a decreasing function of $\sigma$, with

$$
a_{0} \sum_{p \mid k} \frac{\ln (p)}{p-1} \geq D^{*}(k, \sigma) \geq 0 .
$$

We finally need to go from an imprimitive character to a primitive one.

Lemma 3.4.5. The notations being as above, we have

$$
f\left(0, \chi_{0}\right)=f\left(0, \chi^{0}\right)+\sum_{p \mid k} \ln (p) c_{p}(\sigma)
$$

and

$$
f\left(t, \chi_{m}\right) \geq f\left(t, \chi^{m}\right)-\sum_{\substack{p \mid k \\ p \nmid k_{m}}} \ln (p) c_{p}(\sigma) .
$$

Proof. We have for any real number $t$,

$$
f\left(t, \chi_{m}\right)=f\left(t, \chi^{m}\right)+\sum_{\substack{p \mid k \\ p \nmid k_{m}}} \ln (p) \sum_{n \geq 1} \Re\left(\frac{\chi_{m}\left(p^{n}\right)}{p^{i n t}}\right)\left(\frac{1}{p^{n \sigma}}-\frac{\kappa(\sigma)}{p^{n \sigma_{1}}}\right) .
$$

If $\chi_{m}=\chi_{0}$ and $t=0$, then $\chi_{m}\left(p^{n}\right) / p^{i n t}=1$, otherwise its real part is $\geq-1$.

3.5. A positivity argument. The trigonometric polynomial

is also given by

$$
P(\theta)=\sum_{m=0}^{4} a_{m} \cos (m \theta)
$$

$$
P(\theta)=8(0.9126+\cos \theta)^{2}(0.2766+\cos \theta)^{2}
$$

and thus is nonnegative. We have

$$
\sum_{m=0}^{4} a_{m} f\left(m t, \chi^{m}\right)=\sum_{n \geq 1} \Lambda(n)\left(\frac{1}{n^{\sigma}}-\frac{\kappa(\sigma)}{n^{\sigma_{1}}}\right) P\left(\arg \left(\chi(n) n^{-i t}\right)\right) \geq 0 .
$$

Recalling Lemma 3.4.5, we then get

$$
\sum_{m=0}^{4} a_{m} f\left(m t, \chi_{m}\right) \geq a_{0} \sum_{p \mid k} \ln (p) c_{p}(\sigma)-\sum_{m=1}^{4} a_{m} \sum_{\substack{p \mid k \\ p \nmid k_{m}}} \ln (p) c_{p}(\sigma) .
$$


Lemma 3.5.1. We have

$$
a_{0} f\left(0, \chi_{0}\right)+\sum_{m=1}^{4} a_{m}\left(f\left(m t, \chi_{m}\right)+\frac{1-\kappa(\sigma)}{2} \ln \left(\frac{k}{k_{m}}\right)\right) \geq D^{*}(k, \sigma) .
$$

Proof. By the definition of $D^{*}(k, \sigma)$, the inequality in the lemma follows from (3.5.2) if we have

$$
\begin{aligned}
a_{0} \sum_{p \mid k} & \ln (p) c_{p}(\sigma)-\sum_{m=1}^{4} a_{m} \sum_{\substack{p \mid k \\
p \nmid k_{m}}} \ln (p) c_{p}(\sigma) \\
& \geq \sum_{p \mid k} \ln (p) D_{p}^{*}(\sigma)-\sum_{m=1}^{4} a_{m}\left(\frac{1-\kappa(\sigma)}{2}\right) \ln \left(\frac{k}{k_{m}}\right) .
\end{aligned}
$$

However, by Lemma 3.4.3 we have, prime by prime,

$$
a_{0} c_{p}(\sigma)+\sum_{\substack{1 \leq m \leq 4 \\ p \mid \bar{k}, p \nmid k_{m}}} a_{m}\left(\frac{1-\kappa(\sigma)}{2} v_{p}\left(\frac{k}{k_{m}}\right)-c_{p}(\sigma)\right) \geq D_{p}^{*}(\sigma)
$$

which certainly implies (3.5.3).

3.6. The zero-free region. Inserting the bounds from Lemmas 3.3 .1 and 3.3 .3 in the expression from Lemma 3.5.1, if $t$ is taken to be the ordinate of a zero $\beta+i \gamma$ with $\beta>1 / 2$, and $|\gamma| \geq 1000$, we find that $(3.6 .1)$

$$
\begin{aligned}
& a_{0}\left[\frac{1}{\sigma-1}-E(\sigma)\right]+a_{1}\left[\left(\frac{1-\kappa(\sigma)}{2}\right)\left(\ln \left(\frac{k|\gamma|}{2 \pi}\right)+\varepsilon_{1}\right)-\frac{1}{\sigma-\beta}+S(\sigma)\right] \\
& \quad+\sum_{m=2}^{4} a_{m}\left[\left(\frac{1-\kappa(\sigma)}{2}\right)\left(\ln \left(\frac{k_{m}|m \gamma|}{2 \pi}\right)+\varepsilon_{1}\right)+\left(\frac{1-\kappa(\sigma)}{2}\right) \ln \left(\frac{k}{k_{m}}\right)\right] \\
& \quad \geq D^{*}(k, \sigma) .
\end{aligned}
$$

Recalling that $\kappa(\sigma)=\frac{1+\frac{4}{5} \sqrt{\sigma-1}}{\sqrt{5}}$, we can rewrite this as

$$
u-\alpha u \sqrt{\sigma-1}-\frac{w}{\sigma-\beta}+\frac{1}{\sigma-1}-G \geq 0,
$$

where

$$
\left\{\begin{array}{l}
u=\frac{1}{a_{0}} \frac{5-\sqrt{5}}{10} \sum_{m=1}^{4} a_{m}\left(\ln \left(\frac{k m|\gamma|}{2 \pi}\right)+\varepsilon_{1}\right), \\
\alpha=(\sqrt{5}+1) / 5 \cong 0.647213595 \\
w=a_{1} / a_{0} \cong 1.705118356 \\
G=E(\sigma)-w S(\sigma)+\frac{D^{*}(k, \sigma)}{a_{0}} .
\end{array}\right.
$$


Put $x=\sqrt{w}-1 \cong 0.305801806$. A near-optimal value for $\sigma$ in (3.6.2) is $\sigma=1+x / u$. Using this, and the fact that $|\gamma| \geq 1000$, we find that $1 \leq \sigma \leq 1.062$, validating the bounds used in our estimates for $E(\sigma)$ and $S(\sigma)$. Inserting $\sigma=1+x / u$ in (3.6.2) and replacing $G$ by any lower bound $\bar{G}=\bar{G}(\sigma)$, we find

$$
u-\alpha u \sqrt{x / u}-\frac{w}{1-\beta+x / u}+\frac{u}{x}-\bar{G} \geq 0 .
$$

Solving for $1-\beta$ and using $(1+1 / x)=\sqrt{w} / x$ gives

$$
1-\beta \geq \frac{w}{\frac{u \sqrt{w}}{x}-\alpha \sqrt{x u}-\bar{G}}-\frac{x}{u} .
$$

Using $w-\sqrt{w}=x \sqrt{w}$ and cross-multiplying by $u / x^{2}$ yields

$$
\frac{u}{x^{2}}(1-\beta) \geq \frac{1+\frac{\alpha \sqrt{x}}{\sqrt{w u}}+\frac{1}{u \sqrt{w}} \bar{G}}{1-\frac{\alpha x \sqrt{x}}{\sqrt{w u}}-\frac{x}{u \sqrt{w}} \bar{G}} .
$$

Finally, using $x=\sqrt{w}-1$, we obtain

$$
1-\beta \geq \frac{1}{\frac{u}{x^{2}}-\frac{(\alpha / x) \sqrt{u / x}+\left(1 / x^{2}\right) \bar{G}}{1+(\alpha / \sqrt{w}) \sqrt{x / u}+(1 /(x \sqrt{w})(x / u) \bar{G}}} .
$$

Note that

$$
\frac{u}{x^{2}}=R \ln \left(k|\gamma| / C_{1}\right)
$$

where

$$
\left\{\begin{aligned}
R & =\frac{1}{x^{2}} \frac{A}{a_{0}} \frac{5-\sqrt{5}}{10} \cong 9.645908801 \\
C_{1} & =2 \pi \exp \left(-\varepsilon_{1}-(1 / A) \sum_{m=2}^{4} a_{m} \ln (m)\right) \cong 4.171838431 .
\end{aligned}\right.
$$

An especially useful choice for the lower bound $\bar{G}$ in (3.6.4) occurs when $D^{*}(k, \sigma)$ is replaced by a constant lower bound $\bar{D}$, giving

$$
\bar{G}=\bar{G}(\sigma)=E(\sigma)-w S(\sigma)+\frac{\bar{D}}{a_{0}} .
$$

In particular, we can always take $\bar{D}=0$, or, if for some $H \geq 1000$ we are interested only in zeros $\beta+i \gamma$ with $|\gamma| \geq H$, then by Corollary 3.4.4 we can take $\bar{D}$ to be the value of $D^{*}(k, \sigma)$ corresponding to $H$. As will be seen, $\bar{G} \geq 0$ on $[1,1.062]$.

Writing $\Delta=x / u$, and taking $\bar{G}$ as in (3.6.7), put

$$
\begin{aligned}
& G_{1}(\Delta)=\frac{\alpha}{x} \sqrt{\frac{1}{\Delta}}+\frac{1}{x^{2}} \bar{G}(1+\Delta), \\
& G_{2}(\Delta)=1+\frac{\alpha}{\sqrt{w}} \sqrt{\Delta}+\frac{1}{x \sqrt{w}} \cdot \Delta \cdot \bar{G}(1+\Delta) .
\end{aligned}
$$

Then $G_{1}(\Delta) / G_{2}(\Delta)$ is the fraction in the denominator of (3.6.4). 
Lemma 3.6.1. For each $\bar{D} \geq 0$, the functions $G_{1}(\Delta) / G_{2}(\Delta)$ and $\bar{G}(1+\Delta)$ as given by (3.6.7) are nonnegative and decreasing on [0,0.062].

Proof. First suppose $\bar{D}=0$. Note that

$$
E(\sigma)=1.08699+1.40018 \sqrt{\Delta}+1.86576 \Delta+2.32244 \Delta^{3 / 2}
$$

and that (after some manipulations)

$$
S(\sigma)=-\frac{1}{1+\Delta}+\frac{\left(1+\frac{4}{5} \sqrt{\Delta}\right) \sqrt{1-\frac{8}{5} \sqrt{\Delta^{2}+\Delta}+\frac{4}{5}\left(\Delta^{2}+\Delta\right)}}{\left(1-\sqrt{\Delta^{2}+\Delta}\right)^{2}} .
$$

It follows that the derivatives of $\bar{G}(1+\Delta), G_{1}(\Delta)$ and $G_{2}(\Delta)$ are sums and quotients of various terms monotonic on $[0,0.062]$. Thus, on any given subinterval, rigorous upper bounds for $\bar{G}^{\prime}(1+\Delta)$ and $G_{1}^{\prime}(\Delta)$, and a rigorous lower bound for $G_{2}^{\prime}(\Delta)$, can be obtained. Dividing $[0,0.062]$ into subintervals of length 0.001 , it was found that $\bar{G}^{\prime}(1+\Delta)<0$ and $G_{1}^{\prime}(\Delta)<0$ throughout the interval, and $G_{2}^{\prime}(\Delta)>0$ on $[0,0.057]$.

Graphically it is clear that $G_{2}(\Delta)$ has a maximum at about 0.0590 and then slowly decreases. To deal with the interval $[0.057,0.062]$, multiply through by $\sqrt{\Delta}$. The derivatives of $\sqrt{\Delta} \cdot G_{1}(\Delta)$ and $\sqrt{\Delta} \cdot G_{2}(\Delta)$ are sums and quotients of monotonic terms. Dividing $[0,0.062]$ into subintervals of length 0.001 , it was found that $\left(\sqrt{\Delta} \cdot G_{1}(\Delta)\right)^{\prime}<0$ on $[0.047,0.062]$ and $\left(\sqrt{\Delta} \cdot G_{2}(\Delta)\right)^{\prime}>0$ throughout $[0,0.062]$.

Combining these facts yields the monotonicity of $G_{1}(\Delta) / G_{2}(\Delta)$. Its nonnegativity and that of $\bar{G}(1+\Delta)$ follow by evaluation at $\Delta=0.062$.

Now let $\bar{D} \geq 0$ be arbitrary; let $g_{1}(\Delta), g_{2}(\Delta)$ denote $G_{1}(\Delta)$ and $G_{2}(\Delta)$ when $\bar{D}=0$. By the discussion above, it follows that $g_{2} \cdot g_{1}^{\prime}-g_{1} \cdot g_{2}^{\prime}<0$ and $g_{1}^{\prime}<0$ on $[0,0.062]$. Applying the quotient rule to $G_{1}(\Delta) / G_{2}(\Delta)$, it is easily seen that for $G_{1}(\Delta) / G_{2}(\Delta)$ to be decreasing, it suffices to have

$$
g_{2}^{\prime}+\frac{x}{\sqrt{w}} g_{1}>0 .
$$

This was checked by the same means as before.

Theorem 3.6.2. If $\chi$ is a character of conductor $k$, and $R=9.645908801$, then any zero $\beta+i \gamma$ of $L(s, \chi)$ with $|\gamma| \geq 1000$ satisfies

$$
1-\beta \geq \frac{1}{R \ln \left(\frac{k|\gamma|}{4.1718}\right)-3.2356 \sqrt{\ln \left(\frac{k|\gamma|}{4.1718}\right)}} .
$$

Proof. When $\beta>\frac{1}{2}$, the result follows from (3.6.4), taking $\bar{G}=0$ (which is permissible by Lemma 3.6.1); here $3.2356 \leq \alpha \sqrt{R / x} /(1+(\alpha / \sqrt{w}) \sqrt{0.062})$. It is easy to check that the bound is decreasing in $k$ and $|\gamma|$; when $k=1$ and $|\gamma|=1000$, it is less than 0.02194 . Thus the inequality holds when $\beta \leq \frac{1}{2}$ as well.

Taking $\bar{D}=D^{*}(k, \sigma)$ in (3.6.7), we obtain a result better suited to the needs of this paper: recall that $\alpha=(\sqrt{5}+1) / 5, w=a_{1} / a_{0}$ and $x=\sqrt{w}-1$. 
Theorem 3.6.3. If $\chi$ is a character of conductor $k$, and $H \geq 1000$, then any zero $\beta+i \gamma$ of $L(s, \chi)$ with $|\gamma| \geq H$ satisfies

$$
1-\beta \geq \frac{1}{R \ln \left(k|\gamma| / C_{1}(\chi)\right)},
$$

where

$$
C_{1}(\chi)=C_{1}(\chi, H)=C_{1} \cdot \exp \left(\frac{1}{R} \cdot \frac{\frac{\alpha}{x} \sqrt{\frac{1}{\Delta}}+\frac{1}{x^{2}} \bar{G}(1+\Delta)}{1+\frac{\alpha}{\sqrt{w}} \sqrt{\Delta}+\frac{1}{x \sqrt{w}} \cdot \Delta \cdot \bar{G}(1+\Delta)}\right)
$$

with $\Delta=1 /\left(x R \cdot \ln \left(k H / C_{1}\right)\right), \bar{G}$ given by (3.6.7), and $\bar{D}=D^{*}(k, 1+\Delta)$ by (3.4.3).

Proof. When $\beta>\frac{1}{2}$, the result follows from (3.6.4) and Lemma 3.6.1. To see that it holds for $\beta \leq \frac{1}{2}$, one can use the upper bound $11.624 \geq \frac{1}{x^{2}}(E(\sigma)-w S(\sigma))$, the upper and lower bounds for $D^{*}(k, \sigma)$ from Corollary 3.4.4, and the fact that $|\gamma| \geq 1000$ to show that

$$
R \ln \left(k|\gamma| / C_{1}(\chi)\right) \geq 15
$$

\section{Some examples :}

$$
\begin{aligned}
& \text { For } k=1, \quad|\gamma| \geq 545000000, \quad 1-\beta \geq 1 /(R \cdot \ln (k|\gamma| / 38.31)) \text {. } \\
& \text { For } k=3, \quad|\gamma| \geq 10000, \quad 1-\beta \geq 1 /(R \cdot \ln (k|\gamma| / 20.92)) \text {. } \\
& \text { For } k=12, \quad|\gamma| \geq 10000, \quad 1-\beta \geq 1 /(R \cdot \ln (k|\gamma| / 29.68)) \text {. } \\
& \text { For } k=17, \quad|\gamma| \geq 2500, \quad 1-\beta \geq 1 /(R \cdot \ln (k|\gamma| / 20.90)) \text {. } \\
& \text { For } k=420, \quad|\gamma| \geq 2500, \quad 1-\beta \geq 1 /(R \cdot \ln (k|\gamma| / 56.59)) \text {. }
\end{aligned}
$$

4. $L$-FunCtions, $\psi(X ; k, l)$ AND $\theta(X ; k, l)$

Before drawing consequences from Theorem 3.6.3, we need some lemmas.

In the following, we assume that the L-function under examination has no zero satisfying

$$
\left\{\begin{array}{l}
|\gamma| \geq H \geq 1000 \\
\beta>1-\frac{1}{R \ln \left(k|\gamma| / C_{1}(\chi)\right)}
\end{array}\right.
$$

for some positive constants $R$ and $C_{1}(\chi)$ such that

$$
R \cdot \ln \left(\frac{k H}{C_{1}(\chi)}\right) \geq 2 \text {. }
$$

When $R=9.645908801$, then taking $k=1$ and $\gamma=1000$ in Theorem 3.6.2 shows we can always use $C_{1}(\chi)=9.14$; Theorem 3.6.3 gives sharper results.

4.1. Preparatory lemmas. We restate McCurley's results before using them.

First of all, let us note the following consequence of McCurley [5] : 
Lemma 4.1.1 (McCurley). If $\chi$ is a Dirichlet character of conductor $k$, if $T \geq 1$ is a real number, and if $N(T, \chi)$ denotes the number of zeros $\beta+i \gamma$ of $L(s, \chi)$ in the rectangle $0<\beta<1,|\gamma| \leq T$, then

$$
\left|N(T, \chi)-\frac{T}{\pi} \ln \left(\frac{k T}{2 \pi e}\right)\right| \leq C_{2} \ln (k T)+C_{3}
$$

with $C_{2}=0.9185$ and $C_{3}=5.512$.

Our values of $C_{2}$ and $C_{3}$ correspond to the choice $\eta=\frac{1}{2}$ in McCurley's notation and are undoubtedly not optimal. (Note that our $C_{2}$ is his $C_{1}$, and our $C_{3}$ is his $C_{2}$.) If $k>1$, Lemma 4.1.1 follows immediately from ([5, Theorem 2.1]). When $k=1$, it follows from the theorem of Rosser cited in ([5, formula 2.17]).

Lemma 4.1.2. If $k$ is the conductor of $\chi$, and $L(s, \chi)$ satisfies $G R H(H)$ for some $H \geq 1$, then

$$
\sum_{\substack{|\gamma| \leq H \\ \rho \in \mathcal{Z}(\chi)}} \frac{1}{|\rho|} \leq \tilde{E}(H)
$$

with

$$
\tilde{E}(H)=\frac{1}{2 \pi} \ln ^{2}(H)+\frac{\ln \left(\frac{k}{2 \pi}\right)}{\pi} \ln (H)+C_{2}+2\left(\frac{\ln \left(\frac{k}{2 \pi e}\right)}{\pi}+C_{2} \ln (k)+C_{3}\right)
$$

Proof. We consider what happens for $|\gamma| \leq 1$ and for $|\gamma|>1$.

For $|\gamma| \leq 1$, using $\operatorname{GRH}(1)$, we have

$$
\sum_{\substack{|\gamma| \leq 1 \\ \rho \in \mathcal{Z}(\chi)}} \frac{1}{|\rho|} \leq 2 N(1, \chi)
$$

For $|\gamma|>1$, one gets

$$
\begin{aligned}
\sum_{\substack{1<|\gamma| \leq H \\
\rho \in \mathcal{Z}(\chi)}} \frac{1}{|\rho|} \leq \sum_{\substack{1<|\gamma| \leq H \\
\rho \in \mathcal{Z}(\chi)}}\left(\int_{|\gamma|}^{H} \frac{d t}{t^{2}}+\frac{1}{H}\right) \\
=\int_{1}^{H} \frac{N(t, \chi)-N(1, \chi)}{t^{2}} d t+\frac{N(H, \chi)-N(1, \chi)}{H}
\end{aligned}
$$

thus

$$
\sum_{\substack{1<|\gamma| \leq H \\ \rho \in \mathcal{Z}(\chi)}} \frac{1}{|\rho|} \leq \int_{1}^{H} \frac{N(t, \chi)}{t^{2}} d t+\frac{N(H, \chi)}{H}-\frac{N(1, \chi)}{1} .
$$

Now we easily finish the proof on using Lemma 4.1.1. 
Lemma 4.1.3. Let $\chi$ be a character with conductor $k$, let $m \geq 1$ be an integer and $x, x_{0}$ be two real numbers such that $x \geq x_{0} \geq 1$ and $2 R \ln ^{2}\left(k H / C_{1}(\chi)\right) \geq \log \left(x_{0}\right)$. Then

$$
\sum_{\substack{|\gamma| \geq H \\ \rho \in \mathcal{Z}(\chi)}} \frac{x^{\beta}}{|\gamma|^{m+1}}+\sum_{\substack{|\gamma| \geq H \\ \rho \in \mathcal{Z}(\bar{\chi})}} \frac{x^{\beta}}{|\gamma|^{m+1}} \leq x(\tilde{A}+\tilde{B})+\sqrt{x}(\tilde{C}+\tilde{D}),
$$

where, with $C_{2}=0.9185$ and $C_{3}=5.512$, we have

$$
\left\{\begin{array}{l}
\tilde{A}=\int_{H}^{\infty}\left\{\frac{\ln \left(\frac{k t}{2 \pi}\right)}{\pi}+\frac{C_{2}}{t}\right\} \frac{\exp \left\{-\frac{\ln \left(x_{0}\right)}{R \ln \left(k t / C_{1}(\chi)\right)}\right\}}{t^{m+1}} d t, \\
\tilde{B}=\frac{1}{H^{m+1}} \exp \left\{-\frac{\ln \left(x_{0}\right)}{R \ln \left(k H / C_{1}(\chi)\right)}\right\} 2\left(C_{2} \ln (k H)+C_{3}\right), \\
\tilde{C}=\frac{1}{\pi m H^{m}}\left(\ln \left(\frac{k H}{2 \pi}\right)+\frac{1}{m}\right), \\
\tilde{D}=\frac{1}{H^{m+1}}\left(2 C_{2} \ln (k H)+2 C_{3}+\frac{C_{2}}{m+1}\right) .
\end{array}\right.
$$

Proof. The functional equation of the $L$-functions enables us to write

$$
\sum_{\substack{|\gamma| \geq H \\ \rho \in \mathcal{Z}(\chi)}} \frac{x^{\beta}}{|\gamma|^{m+1}}+\sum_{\substack{|\gamma| \geq H \\ \rho \in \mathcal{Z}(\bar{\chi})}} \frac{x^{\beta}}{|\gamma|^{m+1}}=\sum_{\substack{|\gamma| \geq H \\ \rho \in \mathcal{Z}(\chi)}}\left(\frac{x^{1-(1-\beta)}}{|\gamma|^{m+1}}+\frac{x^{1-\beta}}{|\gamma|^{m+1}}\right) .
$$

One of $\beta$ and $1-\beta$ is at most $\frac{1}{2}$, the other is less than

$$
1-\frac{1}{R \ln \left(k|\gamma| / C_{1}(\chi)\right)} .
$$

We can split our upper bound in two pieces. For the first,

$$
\sum_{\substack{|\gamma| \geq H \\ \rho \in \mathcal{Z}(\chi)}} \frac{1}{|\gamma|^{m+1}}
$$

an integration by parts assures that this sum is bounded by $\tilde{C}+\tilde{D}$. For the second,

$$
\tilde{S}(x)=\sum_{\substack{|\gamma| \geq H \\ \rho \in \mathcal{Z}(\chi)}} \frac{x}{|\gamma|^{m+1}} \exp \left\{-\frac{\ln (x)}{R \ln \left(k|\gamma| / C_{1}(\chi)\right)}\right\},
$$

we have $\tilde{S}(x) / x \leq \tilde{S}\left(x_{0}\right) / x_{0}$, hence it remains to evaluate $\tilde{S}\left(x_{0}\right) / x_{0}$. Let us define

$$
\varphi_{m}(t)=\frac{1}{t^{m+1}} \exp \left\{-\frac{\ln \left(x_{0}\right)}{R \ln \left(k t / C_{1}(\chi)\right)}\right\}
$$

and integrate it by parts:

$$
\frac{\tilde{S}\left(x_{0}\right)}{x_{0}}=\sum_{\substack{|\gamma| \geq H \\ \rho \in \mathcal{Z}(\chi)}} \int_{|\gamma|}^{\infty}-\varphi_{m}^{\prime}(t) d t=\int_{H}^{\infty}(N(t, \chi)-N(H, \chi))\left(-\varphi_{m}^{\prime}(t)\right) d t .
$$


But

$$
\frac{\varphi_{m}^{\prime}(t)}{\varphi_{m}(t)}=\frac{m+1}{t \ln ^{2}\left(k t / C_{1}(\chi)\right)}\left[-\ln ^{2}\left(k t / C_{1}(\chi)\right)+\frac{\ln \left(x_{0}\right)}{(m+1) R}\right]
$$

and our hypothesis gives us $-\varphi_{m}^{\prime}(t) \geq 0$ for $t \geq H$.

Lemma 4.1.1 gives us an upper bound for $N(t, \chi)$ and we integrate back by parts.

Lemma 4.1.4. If $k$ is the conductor of $\chi$, and $L(s, \chi)$ satisfies $G R H(1)$, we have

$$
\sum_{\rho \in \mathcal{Z}(\chi)} \frac{2}{|\rho(2-\rho)|} \leq 3.94 \ln (k)+12.7 \text {. }
$$

Proof. The contribution to the sum of the zeros with $|\gamma| \leq 1$ is at most $\frac{8}{3} N(1, \chi)$. The contribution of the remaining ones is not more than

$$
4 \int_{1}^{\infty} \frac{N(t, \chi)-N(1, \chi)}{t^{3}} d t=-2 N(1, \chi)+4 \int_{1}^{\infty} \frac{N(t, \chi)}{t^{3}} d t
$$

and on using Lemma 4.1.1, we obtain the result.

4.2. Computing $\tilde{A}$. The constant $\tilde{A}$ is the dominant term in Lemma 4.1.3. However, the integral defining $\tilde{A}$ converges slowly, and care is needed in evaluating it. This was forcefully brought home to us when we initially sought to compute $\tilde{A}$ using the pre-programmed numerical integration of PARI. Lionel Reboul of the University of Lyon kindly verified the values by independent computations with MAPLE. To our surprise, the values were the same for $\ln \left(x_{0}\right) \geq 40$, but they were not close for small $x_{0}$ (for instance 0.032 instead of 0.034 ).

We will first give a simple lemma (Lemma 4.2.1) which gives a rather good upper bound for $\tilde{A}$. Then we will describe a way for accurately computing $\tilde{A}$ advocated by Rosser, Schoenfeld, and McCurley, which involves transforming the given integral to a sum of rapidly convergent incomplete Bessel integrals.

For $m \geq 2$ let us define $h_{m}(t)$ by

$$
h_{m}(t)=\frac{1}{\pi(m-1) t^{m-1}}\left(\ln \left(\frac{k t}{2 \pi}\right)+\frac{1}{m-1}\right)+\frac{C_{2}}{m t^{m}} .
$$

Lemma 4.2.1. We have, under the hypothesis of Lemma 4.1.3, but with in addition $R \ln ^{2}\left(k H / C_{1}(\chi)\right) \geq \ln \left(x_{0}\right)$ and $m \geq 2$,

$$
\left\{\begin{array}{l}
\tilde{A} \leq \frac{h_{m}(H)}{H} \exp \left(-\frac{\ln \left(x_{0}\right)}{R \ln \left(k H / C_{1}(\chi)\right)}\right) \\
\tilde{A} \geq h_{m+1}(H) \exp \left(-\frac{\ln \left(x_{0}\right)}{R \ln \left(k H / C_{1}(\chi)\right)}\right) .
\end{array}\right.
$$

Proof. Let us define

$$
g_{m}(t)=\frac{\ln \left(\frac{k t}{2 \pi}\right)}{\pi t^{m}}+\frac{C_{2}}{t^{m+1}} .
$$


Then, we readily see that $h_{m}^{\prime}(t)=-g_{m}(t)$. We also have

$$
\begin{aligned}
\tilde{A}= & \int_{H}^{\infty} g_{m}(t) \exp \left(-\frac{\ln \left(x_{0}\right)}{R \ln \left(k t / C_{1}(\chi)\right)}\right) \frac{d t}{t} \\
= & \frac{h_{m}(H)}{H} \exp \left(-\frac{\ln \left(x_{0}\right)}{R \ln \left(k H / C_{1}(\chi)\right)}\right) \\
& -\int_{H}^{\infty}\left(\ln ^{2}\left(k t / C_{1}(\chi)\right)-\frac{\ln \left(x_{0}\right)}{R}\right) \exp \left(-\frac{\ln \left(x_{0}\right)}{R \ln \left(k t / C_{1}(\chi)\right)}\right) \frac{h_{m}(t) d t}{t^{2} \ln ^{2}\left(k t / C_{1}(\chi)\right)} \\
\leq & \frac{h_{m}(H)}{H} \exp \left(-\frac{\ln \left(x_{0}\right)}{R \ln \left(k H / C_{1}(\chi)\right)}\right)
\end{aligned}
$$

the last inequality holds because our hypothesis ensures that

$$
\left(\ln ^{2}\left(k H / C_{1}(\chi)\right)-\frac{\ln \left(x_{0}\right)}{R}\right) \geq 0 .
$$

For the other inequality, we write

$$
\tilde{A}=\int_{H}^{\infty} g_{m+1}(t) \exp \left(-\frac{\ln \left(x_{0}\right)}{R \ln \left(k t / C_{1}(\chi)\right)}\right) d t
$$

and an integration by parts yields the result.

We now turn to the accurate computation of $\tilde{A}$. Define

$$
\left\{\begin{array}{l}
K_{n}(z, w)=\frac{1}{2} \int_{w}^{\infty} u^{n-1} \exp \left[-\frac{z}{2}\left(u+\frac{1}{u}\right)\right] d u \\
U_{m}=\sqrt{\frac{R m}{\ln \left(x_{0}\right)}} \ln \left(\frac{k H}{C_{1}(\chi)}\right), \\
z_{m}=2 \sqrt{\frac{m \ln \left(x_{0}\right)}{R}}
\end{array}\right.
$$

Then simple algebraic manipulations yield

Lemma 4.2.2. There holds

$$
\begin{aligned}
\tilde{A}= & \frac{2}{\pi} \frac{\ln \left(x_{0}\right)}{R m}\left(\frac{k}{C_{1}(\chi)}\right)^{m} K_{2}\left(z_{m}, U_{m}\right) \\
& +\frac{2}{\pi} \ln \left(\frac{C_{1}(\chi)}{2 \pi}\right) \sqrt{\frac{\ln \left(x_{0}\right)}{R m}}\left(\frac{k}{C_{1}(\chi)}\right)^{m} K_{1}\left(z_{m}, U_{m}\right) \\
& +2 C_{2} \sqrt{\frac{\ln \left(x_{0}\right)}{R(m+1)}}\left(\frac{k}{C_{1}(\chi)}\right)^{m+1} K_{1}\left(z_{m+1}, U_{m+1}\right) .
\end{aligned}
$$

The tails in the integrals $K_{1}$ and $K_{2}$ can be estimated as follows. Write

$$
\operatorname{erfc}(x)=\frac{2}{\sqrt{\pi}} \int_{x}^{\infty} e^{-t^{2}} d t .
$$


Lemma 4.2.3 (Rosser and Schoenfeld). Put $y=\frac{\sqrt{w}-1 / \sqrt{w}}{\sqrt{2}}$ for $w \geq 1$. Then

$$
K_{1}(z, w)<\frac{e^{-z}}{2 z}\left\{\left(1+\frac{3 \sqrt{2}}{8} y\right) e^{-z y^{2}}+\left(\frac{3}{8 \sqrt{z}}+\sqrt{z}\right) \sqrt{\frac{\pi}{2}} \operatorname{erfc}(y \sqrt{z})\right\},
$$

and

$$
\begin{aligned}
K_{2}(z, w)<\frac{e^{-z}}{2 z}\left\{\left[\frac{35 \sqrt{2}}{64} y^{3}+2 y^{2}\right.\right. & \left.+\left(\frac{105}{128 z}+\frac{15}{8}\right) \sqrt{2} y+2+\frac{2}{z}\right] e^{-z y^{2}} \\
& \left.+\left(\frac{105}{128 z}+\frac{15}{8}+z\right) \sqrt{\frac{\pi}{2 z}} \operatorname{erfc}(y \sqrt{z})\right\} .
\end{aligned}
$$

Lemma 4.2.4. Assume $z>0$.

The integrand in $K_{1}(z, w)$ is decreasing in $u$ for $u \geq 1$.

The integrand in $K_{2}(z, w)$ is increasing for $1 \leq u \leq \frac{1+\sqrt{1+z^{2}}}{z}$ and decreasing in $u$ afterwards.

Lemma 4.2 .3 is accurate if $w$ is large. Thus, $K_{1}$ and $K_{2}$ can be computed by integrating numerically from $w$ to some finite bound and then using Lemma 4.2.3. In computing Table 1, this method was used in conjunction with Simpson's rule to get a sharp rigorous upper bound for $\tilde{A}$ (within $1 \%$ ). It should also be noted that R. Terras [13] has given a rapid continued fraction algorithm for computing integrals like $K_{1}$ and $K_{2}$. Although this algorithm does not produce a rigorous error bound, we have used it to give an independent check on the numerical integrations.

4.3. Estimating $\psi$ and $\theta$ through $L$-functions. We are now in a position to prove Theorem 1. To do so we want to use Theorem 3.6 of McCurley [5]. But in that theorem, the notation $\mathcal{Z}(\chi)$ denotes the set of zeros of $L(s, \chi)$ with $0 \leq \beta<1$, so it is necessary to pay careful attention to the distinction between conductor and modulus. In [5], the zeros with $\beta=0$ are removed with some cumbersome arguments (cf. [5, (3.25), (3.26), (3.27) and (3.38)]). The troubles caused by the use of imprimitive characters can also be seen in ([5, (3.15)]).

However, it is possible to work from the beginning with primitive characters only. If $\chi$ is a character modulo $k$, let $\chi_{1}$ be its associated primitive character. Given $l$ prime to $k$, consider further

$$
w_{k}(l, n)=\frac{1}{\varphi(k)} \sum_{\chi \bmod k} \chi_{1}(n) \bar{\chi}(l) .
$$

If $K$ is the largest divisor of $k$ coprime to $n$, we have

$$
w_{k}(l, n)= \begin{cases}\frac{\varphi(K)}{\varphi(k)} & \text { if } n \equiv l(\bmod K) \\ 0 & \text { otherwise }\end{cases}
$$

which can be proved as follows:

$$
\begin{aligned}
\varphi(k) w_{k}(l, n) & =\sum_{d \mid k} \sum_{\chi \bmod ^{*} d} \chi(n) \bar{\chi}(l) \\
& =\sum_{d \mid K} \sum_{\chi \bmod ^{*} d} \chi(n) \bar{\chi}(l)=\sum_{\chi \bmod K} \chi(n) \bar{\chi}(l),
\end{aligned}
$$


where $\sum_{\chi} \bmod { }^{*} d$ is a summation over the primitive characters modulo $d$. We now consider

$$
\psi^{*}(x ; k, l)=\sum_{n \leq x} w_{k}(l, n) \Lambda(n) .
$$

We have $\psi(x ; k, l) \leq \psi^{*}(x ; k, l) \leq \psi(x ; k, l)+f(k) \ln (x)$ for $x \geq 1$ with

$$
f(k)=\sum_{p \mid k} \frac{1}{p-1} .
$$

We remark that $f(k) \leq 3.5$ if $k$ has less than 12000 prime factors, which is true if $k \leq$ $\exp (127000)$, and in any case $f(k)=\mathcal{O}(\ln (\ln (\ln (k))))$. Thus, we can work with $\psi^{*}$ instead of $\psi$ with a small loss. Now McCurley's analysis ([5, §3]) goes through with characters replaced by primitive characters since $\psi^{*}$ is also an increasing function. More precisely, McCurley's $m(\chi)$ now becomes the order of the zero of $L\left(s, \chi_{1}\right)$ at $s=0$, i.e. $m(\chi)=0$ if $\chi(-1)=-1$ or $\chi$ is the principal character, and $m(\chi)=1$ otherwise. In McCurley's notation, this yields $\left|d_{2}\right| \leq 1 / 2$. Following the proof of Lemma 3.5 of [5], we find further that if $L(s, \chi)$ satisfies $\operatorname{GRH}(1)$ and $\chi$ is not principal, then

$$
|b(\chi)| \leq 1.57+\sum_{\rho \in \mathcal{Z}(\chi)} \frac{2}{|\rho(2-\rho)|},
$$

which, with Lemma 4.1.4 together with $\left|b\left(\chi_{0}\right)\right|=\ln (2 \pi)$ yields "If every $L$-function of modulus $k$ satisfies GRH(1), then $\left|d_{1}+d_{2}\right| \leq 3.94 \ln (k)+12.7$ " instead of Lemma 3.5 of [5]. Finally, the right-hand side of $([5,(3.21)])$ becomes

$$
(\delta x)^{m}\left[\frac{\ln (2)}{2}+\frac{\ln (2 x)}{2}+3.94 \ln (k)+12.7\right] .
$$

An additional error term comes from replacing $\psi^{*}$ by $\psi$. The upper bound we get this way is an increasing function of $x$, so we can introduce the maximum $\max _{1 \leq y \leq x}$ which is useful in practice. Thus we get

Theorem 4.3.1 (McCurley). Let $x \geq 1$ be a real number, $k \geq 1$ an integer, $m a$ positive integer, $\delta$ a real number with $0<\delta<\frac{x-2}{m x}$, and $H$ a positive real number. Put

$$
A(m, \delta)=\frac{1}{\delta^{m}} \sum_{j=0}^{m}\left(\begin{array}{c}
m \\
j
\end{array}\right)(1+j \delta)^{m+1} .
$$

Suppose that for each $\chi$ with modulus $k, L(s, \chi)$ satisfies $G R H(1)$. Then

$$
\begin{aligned}
\frac{\varphi(k)}{x} \max _{1 \leq y \leq x}\left|\psi(y ; k, l)-\frac{y}{\varphi(k)}\right|< & A(m, \delta) \sum_{\chi} \sum_{\substack{\rho \in \mathcal{Z}(\chi) \\
|\gamma|>H}} \frac{x^{\beta-1}}{|\rho(\rho+1) \ldots(\rho+m)|} \\
& +\left(1+\frac{m \delta}{2}\right) \sum_{\substack { \chi \\
\begin{subarray}{c}{\rho \in \mathcal{Z}(\chi) \\
|\gamma| \leq H{ \chi \\
\begin{subarray} { c } { \rho \in \mathcal { Z } ( \chi ) \\
| \gamma | \leq H } }\end{subarray}} \frac{x^{\beta-1}}{|\rho|}+\frac{m \delta}{2}+\tilde{R},
\end{aligned}
$$

where $\sum_{\chi}$ denotes the summation over all characters modulo $k$,

$$
\tilde{R}=\frac{\varphi(k)}{x}[(f(k)+0.5) \ln (x)+4 \ln (k)+13.4],
$$

and $f(k)$ is given by (4.3.1). 
We also wish to allow $\theta$ instead of $\psi$ which can be done by recalling Theorem 6 of Rosser and Schoenfeld ([9]) :

$$
0 \leq \psi(x ; k, l)-\theta(x ; k, l) \leq \psi(x)-\theta(x) \leq 1.0012 \sqrt{x}+3 x^{\frac{1}{3}} \text { for } x>0 .
$$

Collecting our results, we finally get

Theorem 4.3.2. Let $k \geq 1$ be an integer, and let $H \geq 1000$ be a real number such that every L-function with modulus $k$ satisfies $G R H(H)$. Put $C=C_{1}\left(\chi_{0}, H\right)$, where $\chi_{0}$ is the trivial character, and let $x_{0}$ be a real number such that $R \cdot \ln ^{2}(H / C) \geq$ $\ln \left(x_{0}\right)$. Let $m \geq 2$ be an integer, and let $\delta>0$ be a real number such that $0<\delta<$ $\left(x_{0}-2\right) /\left(m x_{0}\right)$.

Then for any $x \geq x_{0}$ we have

$$
\begin{aligned}
\frac{\varphi(k)}{x} \max _{1 \leq y \leq x}\left|\psi(y ; k, l)-\frac{y}{\varphi(k)}\right| \leq & \frac{\left(1+(1+\delta)^{m+1}\right)^{m}}{\delta^{m}} \frac{\varphi(k)}{2}\left((\tilde{A}+\tilde{B})+\frac{1}{\sqrt{x_{0}}}(\tilde{C}+\tilde{D})\right) \\
& +\left(1+\frac{m \delta}{2}\right) \frac{\varphi(k)}{\sqrt{x_{0}}} \tilde{E}(H)+\frac{m \delta}{2}+\tilde{R},
\end{aligned}
$$

where $\tilde{A}, \tilde{B}, \tilde{C}, \tilde{D}$ are those of Lemma 4.1 .3 with $C_{1}(\chi)$ replaced by $C$ and with $\tilde{A}$ estimated by Lemma 4.2.1, $\tilde{E}(H)$ given by Lemma 4.1.2, and $\tilde{R}$ given by (4.3.2) with $x=x_{0}$.

If $\tilde{\Delta}=\varphi(k)\left(1.0012 x_{0}^{-1 / 2}+3 x_{0}^{-2 / 3}\right)$, and $\tilde{Z}$ denotes the error bound above, then $\tilde{Z}+\tilde{\Delta}$ is an upper bound for

$$
\frac{\varphi(k)}{x} \max _{1 \leq y \leq x}\left|\theta(y ; k, l)-\frac{y}{\varphi(k)}\right| .
$$

Proof. We use Theorem 4.3.1 and evaluate the sums over the zeros by appealing to Lemmas 4.1.2 and 4.1.3. We also remark (cf. [8]) that

$$
A(m, \delta) \leq \frac{\left(1+(1+\delta)^{m+1}\right)^{m}}{\delta^{m}}
$$

Theorem 4.3.2 has been stated in such a way as to make its implementation as simple as possible, and to minimize the numerical input required. For a stronger version which yields slightly better results, see Theorem 5.1 .1 below.

4.4. Heuristic control of the values of $m, \delta$ and $\varepsilon$. Let $\varepsilon_{k}(m, \delta)$ denote the expression $\tilde{Z}+\tilde{\Delta}$ in Theorem 4.3 .2 , and let $\varepsilon_{k}$ be its minimum value as $m$ and $\delta$ vary. This optimization is achieved in practice by first minimizing over $\delta$ (with $m$ held fixed) using any standard minimization algorithm, and then minimizing over $m$.

In this section we aim to heuristically estimate the optimal values of $m$ and $\delta$ and the order of magnitude of $\varepsilon_{k}$, together with their dependence on $k$ and $H$. Under the hypothesis of Theorem 4.3.2, we note that in the formula for $\tilde{Z}+\tilde{\Delta}$ :

(1) $\left(1+(1+\delta)^{m+1}\right)^{m} / \delta^{m}$ is equivalent to $2^{m} / \delta^{m}$ if $m \delta$ tends to 0 . 
(2) $\left(\tilde{A}+\tilde{B}+(\tilde{C}+\tilde{D}) / \sqrt{x_{0}}\right)$ can be approximated by $\ln \left(\frac{k H}{2 \pi}\right) /\left(\pi m H^{m}\right)$ for large $H$ and $x_{0}$.

(3) $(1+m \delta / 2) \tilde{E}(H) x_{0}^{-1 / 2}$ is asymptotic to $x_{0}^{-1 / 2} \frac{\ln ^{2}(k H)}{2 \pi}$ as $H$ goes to infinity and $\delta$ to 0 . For large $x_{0}$ it is negligible.

(4) $\tilde{R}$ is $\mathcal{O}\left(k \ln \left(k x_{0}\right) / x_{0}\right)$ when $x_{0}$ goes to infinity. For $x_{0}$ large it is negligible.

(5) For large $x_{0}, \tilde{\Delta}$ is negligible.

Hence our $\varepsilon_{k}$ can be approximated by

$$
f(m, \delta)=\frac{m \delta}{2}+\frac{\varphi(k)}{2}\left(\frac{2}{\delta H}\right)^{m} \frac{\ln \left(\frac{k H}{2 \pi}\right)}{\pi m} .
$$

We reduce this expression once more; numerically, it happens that the values of $m$ are almost always near 7 . We replace the term $\left(\frac{2}{\delta H}\right)^{m} \frac{1}{m}$ by $\left(\frac{2}{\delta H}\right)^{m} \frac{1}{\bar{m}}$, where $\bar{m}$ is an estimation of the value of $m$ and we finally consider

$$
\bar{f}(m, \delta)=\frac{a}{(\delta B)^{m}}+\frac{m \delta}{2}
$$

with

$$
a=\frac{\varphi(k)}{2} \frac{\ln \left(\frac{k H}{2 \pi}\right)}{\pi \bar{m}} \text { and } \quad B=\frac{H}{2} .
$$

We now let $m$ vary continuously. Then

$$
\frac{\partial \bar{f}}{\partial \delta}=\frac{\partial \bar{f}}{\partial m}=0
$$

yields

$$
\left\{\begin{array}{l}
\frac{a}{(\delta B)^{m}}=\frac{\delta}{2}, \\
\frac{a}{(\delta B)^{m}} \ln (\delta B)=\frac{\delta}{2},
\end{array}\right.
$$

hence

$$
\begin{gathered}
\delta=\frac{2 e}{H}, \quad m=\ln (a H / e) \\
\bar{f}(m, \delta)=(m+1) \frac{\delta}{2}=\ln \left(\frac{H \varphi(k)}{2 \pi \bar{m}} \ln \left(\frac{k H}{2 \pi}\right)\right) \cdot \frac{e}{H} .
\end{gathered}
$$

It is striking that in this approximation, $\delta$ depends neither on $m$ nor on $k$. We have verified this numerically ( $\delta$ is almost constant). The other striking thing is that $\bar{f}(m, \delta)$ (our $\varepsilon_{k}$ ) depends on $k$ mainly through $\ln (\varphi(k)$ ); we find numerically that our $\varepsilon_{k}$ 's increase very slowly when $k$ increases. This is rather surprising since there are $\varphi(k)$ sums to evaluate, so we might have expected $\varepsilon_{k}$ to depend on $k$ by a multiplicative factor $\varphi(k)$. 


\section{Numerical EStimates FOR $\theta$ AND $\psi$}

In this section, we present the numerical estimates one can deduce from the results in $\S \S 2,3$, and 4 . Since the analytic bounds only become useful for $x_{0} \geq 10^{10}$, we have supplemented them with a tabulation for low heights.

The following notation is used throughout:

$$
\left\{\begin{array}{l}
\varepsilon(\theta, x, k)=\max _{(l, k)=1} \frac{\varphi(k)}{x} \max _{1 \leq y \leq x}|\theta(y ; k, l)-y / \varphi(k)|, \\
\varepsilon(\psi, x, k)=\max _{(l, k)=1} \frac{\varphi(k)}{x} \max _{1 \leq y \leq x}|\psi(y ; k, l)-y / \varphi(k)| .
\end{array}\right.
$$

The computations for the tables were performed on PCs using the Intel 80486 processor. The 80486-DX was used in Extended Precision mode, where it calculates with an 80-bit word having a 1-bit sign, a 15-bit exponent, and a 64-bit mantissa. It carries approximately 19.2 decimal digits of accuracy, and can represent numbers over the range $10^{-4392}$ to $10^{4392}$. In Tables 1 and 2 , the results are reported to 6 digits of accuracy, and were rounded up in their last digit.

As a verification, independent computations were done using the calculator GP of PARI, and some randomly chosen values were checked, with satisfying agreement.

\subsection{Asymptotic results for $x_{0} \geq 10^{10}$.}

Theorem 1. For any triple $\left(k, \varepsilon, x_{0}\right)$ given by Table 1 ,

$$
\left\{\begin{array}{l}
\varepsilon(\theta, x, k) \leq \varepsilon \text { for } x \geq x_{0} \\
\varepsilon(\psi, x, k) \leq \varepsilon \text { for } x \geq x_{0}
\end{array}\right.
$$

Table 1 contains the best known bounds for all the moduli covered by Rumely's $L$-series calculations. The bounds were computed using a strong form of Theorem 4.3.2, which broke the error term into its contributions from each individual $L$ series, and replaced the estimates for $\tilde{E}(H)$ by tabulated values:

Theorem 5.1.1. Let $k \geq 1$ be an integer. For each character $\chi$ modulo $k$, let $H_{\chi} \geq 1000$ be such that $L(s, \chi)$ satisfies $G R H\left(H_{\chi}\right)$; we suppose $H_{\chi}=H_{\bar{\chi}}$ for all $\chi$, and put $C_{1}(\chi)=C_{1}\left(\chi, H_{\chi}\right)$. Let $x_{0} \geq 10$ be a real number such that for each $d$ dividing $k$, and each $\chi$ with conductor $d, 2 R \cdot \ln ^{2}\left(d H_{\chi} / C_{1}(\chi)\right) \geq \ln \left(x_{0}\right)$. Let $m$ be a positive integer, and let $\delta>0$ be a real number such that $0<\delta<\left(x_{0}-2\right) /\left(m x_{0}\right)$. For each $\chi$ with conductor $d$, let $\tilde{A}_{\chi}, \tilde{B}_{\chi}, \tilde{C}_{\chi}, \tilde{D}_{\chi}$ be the constants from Lemma 4.1.3 computed using $d$ and $H_{\chi}$; let $\tilde{E}_{\chi}$ be a tabulated bound for $\sum_{\substack{\rho \in Z(\chi) \\|\beta| \leq H_{\chi}}} 1 /|\rho|$. Let $\tilde{R}$ be as in Theorem 4.3.1.

Then for $x \geq x_{0}$

$$
\begin{aligned}
\varepsilon(\psi, x, k) \leq \frac{1}{2} A(m, \delta) & \cdot \sum_{\chi}\left[\tilde{A}_{\chi}+\tilde{B}_{\chi}+\left(\tilde{C}_{\chi}+\tilde{D}_{\chi}\right) / \sqrt{x_{0}}\right] \\
& +\frac{(1+m \delta / 2)}{\sqrt{x_{0}}} \cdot\left(\sum_{\chi} \tilde{E}_{\chi}\right)+m \delta / 2+\tilde{R} .
\end{aligned}
$$


TABLE 1. Analytic epsilons for $x \geq x_{0}$

The values reported are $\varepsilon=\max \left(\varepsilon\left(\theta, x_{0}, k\right), \varepsilon\left(\psi, x_{0}, k\right)\right)$, and have been rounded up in the last decimal place (our notation is as in (5.1) of the paper).

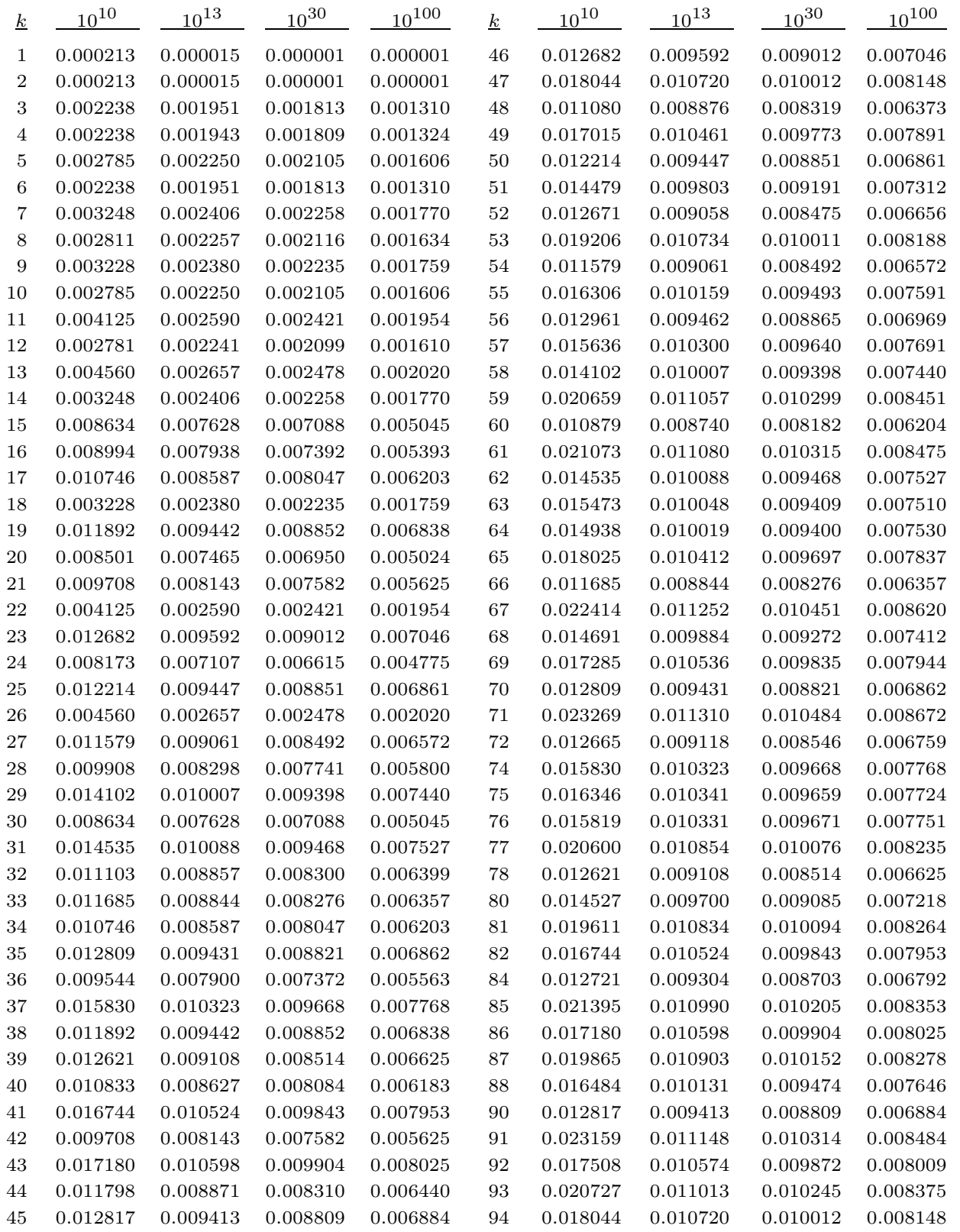


TABLE 1 (continued)

\begin{tabular}{|c|c|c|c|c|c|c|c|c|c|}
\hline$\underline{k}$ & $10^{10}$ & $10^{13}$ & $10^{30}$ & 100 & $\underline{k}$ & $10^{10}$ & $10^{13}$ & $10^{30}$ & $0^{100}$ \\
\hline 95 & 0.023180 & 1282 & 0.010444 & 0.008570 & 144 & 0.018241 & 0.010486 & .009772 & 0.00797 \\
\hline 96 & & & 271 & & 150 & & & & \\
\hline 98 & 17015 & 1 & 0.009773 & 1 & 154 & 00 & & & \\
\hline 99 & 44 & & & & 66 & & & & \\
\hline 0 & 476 & & & & 162 & & & & 264 \\
\hline 20 & .014479 & 009803 & 0.009191 & 12 & 63 & & & 11505 & 09799 \\
\hline 4 & 292 & 424 & & & 38 & & & 40 & 07882 \\
\hline 55 & & & & & & & & & \\
\hline 6 & 06 & 34 & & & 170 & & & & 353 \\
\hline & 35 & 79 & 41 & 39 & 174 & & & & 278 \\
\hline & 06 & 59 & 93 & & 180 & & & 63 & 903 \\
\hline & 87 & & 0.0 & & & & & & 484 \\
\hline 12 & & & & & & & & & 375 \\
\hline 14 & & & & & & & & & 570 \\
\hline 16 & & & & & & & & & 238 \\
\hline 17 & & & & & 10 & & & & \\
\hline 18 & & & & & 216 & & & & \\
\hline 20 & & & & & 222 & & & & \\
\hline 21 & & & & & & & & & 499 \\
\hline 22 & 0.021073 & 0.0 & & & 24 & & & & 223 \\
\hline 24 & 0021 & 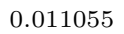 & & & & & & & 9740 \\
\hline 25 & 29581 & 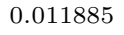 & & & 2 & & & 860 & .009072 \\
\hline 26 & 015473 & 0048 & 09 & 10 & 256 & & & 1179 & .009461 \\
\hline 28 & 022035 & $0 .($ & 36 & & 286 & & & 952 & 0.009218 \\
\hline & & & & & & & & 505 & 0.009799 \\
\hline 3 & & & & & & & & 11383 & 0.009670 \\
\hline 32 & & & & & & & & 644 & 0.008854 \\
\hline 38 & 85 & 0.010536 & & & 420 & & 0.011602 & .010617 & 0.008766 \\
\hline 140 & 0.017989 & 0.010523 & 0.009796 & 0.007898 & 432 & 0.039660 & 0.012542 & .011234 & 0.009519 \\
\hline 142 & 269 & 0.011310 & 010484 & 0.008672 & 486 & 0.043836 & 0.012879 & 0.011458 & 0.009740 \\
\hline
\end{tabular}
then

If $\tilde{\Delta}=\varphi(k)\left(1.0012 x_{0}^{-1 / 2}+3 x_{0}^{-2 / 3}\right)$, and $\tilde{Z}$ denotes the above bound for $\varepsilon(\psi, x, k)$,

$$
\varepsilon(\theta, x, k) \leq \tilde{Z}+\tilde{\Delta}
$$

If $k$ is not of the form $k=2 k^{\prime}$ with $k^{\prime}$ odd, and $k \neq 1$, then the values reported in Table 1 are the minimal values of the expression $\tilde{Z}+\tilde{\Delta}$ in Theorem 5.1.1, when $2 \leq m \leq 14$ and $\delta$ ranges over its allowable values. The minima were found using Brent's golden section/parabolic interpolation algorithm ([7, p. 283]). It should be noted that as $x_{0}$ and the $H_{\chi}$ vary, different terms in the expression become dominant. Heuristically the best estimate is obtained using GRH data to height $H \cong 4 e \pi \cdot \sqrt{x_{0}} /\left(\varphi(k) \cdot \ln \left(x_{0}\right)\right)$. The values in Table 1 are minima over the following data sets:

(A) Rumely's $L$-series and zeta data, using $T=2500$ data for $k \leq 13$,

(B) Rumely's $L$-series and zeta data, using $T=5000$ data for $k \leq 13$,

(C) Rumely's $L$-series and zeta data,

(D) Rumely's $L$-series data + Lehman's zeta data, with $T=12030$, 
(E) Rumely's $L$-series data + vdL, teR, Winter zeta data to $T=10^{5}$,

(F) Rumely's $L$-series data $+\mathrm{vdL}$, teR, Winter zeta data to $T=10^{6}$,

(G) Rumely's $L$-series data $+\mathrm{vdL}$, teR, Winter zeta data to $T=10^{7}$,

$(\mathrm{H})$ Rumely's $L$-series data $+\mathrm{vdL}$, teR, Winter zeta data to $T=545439823$.

If $k=2 k^{\prime}$ with $k^{\prime}$ odd, and if $N$ is the order of 2 in $\left(\mathbb{Z} / k^{\prime} \mathbb{Z}\right)^{\times}$, then for $(k, l)=1$

$$
\begin{gathered}
\left|\psi(x ; k, l)-\psi\left(x ; k^{\prime}, l\right)\right| \leq(1 / N) \ln (x)+\ln (2), \\
\left|\theta(x ; k, l)-\theta\left(x ; k^{\prime}, l\right)\right| \leq \ln (2) .
\end{gathered}
$$

For the values of $x_{0}$ considered in Table 1 , these inequalities yield estimates for $\varepsilon(\theta, x, k), \varepsilon(\psi, x, k)$ better than those provided by Theorem 5.1.1 directly, and are the ones reported. It will be observed that the bounds for $k$ and $k^{\prime}$ in Table 1 are always the same: the additional term in the error estimate was swallowed up when the values were rounded up to the nearest $10^{-6}$.

Finally, when $k=1,2$ the $x_{0}=10^{10}$ entry in Table 1 is derived from the main table of Rosser and Schoenfeld ([9]), which gives $\varepsilon\left(\psi, e^{23}, 1\right)=0.00020211$; this improves on the value 0.000272 given by Theorem 5.1.1.

5.2. Estimates over the range $0-10^{10}$. In Table 2 we compare $\mid \psi(x ; k, l)-$ $x / \varphi(k) \mid$ and $|\theta(x ; k, l)-x / \varphi(k)|$ with $\sqrt{x}$, reporting the maximum ratio for $0<$ $x \leq 10^{10}$ for each $k$. Tabulations over the subintervals $\left[10^{n}, 10^{n+1}\right]$ show that the results are remarkably stable and uniform over the entire range, so that Table 2 gives a good representation of the true error. The only exception is for very small moduli $(k \leq 6)$ where the initial primes distort the error estimates; more accurate asymptotic values are given at the end of the table for these moduli. Let $N(k, l)$ be the number of solutions to $a^{2} \equiv l(\bmod k)$ with $0 \leq a<k$. The function $(x-N(k, l) \sqrt{x}) / \varphi(k)$ usually gives a better approximation to $\theta(x ; k, l)$ than $x / \varphi(k)$; we have also tabulated the maximal ratios $|\theta(x ; k, l)-(x-N(k, l) \sqrt{x}) / \varphi(k)| \sqrt{x}$.

We can summarize the main results of Table 2 as follows:

Theorem 5.2.1. For all the moduli in Table 1, uniformly over the range $0 \leq x \leq$ $10^{10}$, the following bounds hold:

$$
\begin{aligned}
|\theta(x ; k, l)-x / \varphi(k)| & \leq 2.072 \sqrt{x}, \\
\left|\theta(x ; k, l)-\frac{x-N(k, l) \sqrt{x}}{\varphi(k)}\right| & \leq 1.174 \sqrt{x}, \\
|\psi(x ; k, l)-x / \varphi(k)| & \leq 1.745 \sqrt{x} .
\end{aligned}
$$

If $k=5, k \geq 7$ or $x \geq 224$, then

$$
|\psi(x ; k, l)-x / \varphi(k)| \leq \sqrt{x}
$$

for $k=1,3,4$ this holds if $x \geq 14$.

Corollary 5.2.2. For these moduli, over the range $0<x \leq 10^{10}$,

$$
\varepsilon(\theta, x, k) \leq 2.072 \frac{\varphi(k)}{\sqrt{x}}, \quad \varepsilon(\psi, x, k) \leq 1.745 \frac{\varphi(k)}{\sqrt{x}} ;
$$

for $k=5$ or $k \geq 7, \varepsilon(\psi, x, k) \leq \varphi(k) / \sqrt{x}$. 
TABLE 2. Tabulations $0-10^{10}$

$k:$ The modulus.

$\theta: \max _{(l, k)=1} \max _{\left(0,10^{10}\right]}|\theta(x ; k, l)-x / \varphi(k)| / \sqrt{x}$

$\theta \#: \max _{(l, k)=1} \max _{\left(0,10^{10}\right]}|\theta(x ; k, l)-(x-N(k, l) \sqrt{x}) / \varphi(k)| / \sqrt{x}$,

(1) where $N(k, l)$ is the number of solutions to $a^{2} \equiv l(\bmod k)$

$\psi: \max _{(l, k)=1} \max _{\left(0,10^{10}\right]}|\psi(x ; k, l)-x / \varphi(k)| / \sqrt{x}$

Except for the $\psi$-values for a few small moduli, the bounds for $\theta, \theta \#$ and $\psi$ are remarkably good throughout the range $0-10^{10}$. All values have been rounded up in the last decimal place.

\begin{tabular}{|c|c|c|c|c|c|c|}
\hline$\theta$ & $\theta \#$ & $\psi$ & $\underline{k}$ & $\theta$ & $\theta \#$ & $\psi$ \\
\hline 2.052818 & 1.052818 & $1.414214^{*}$ & 39 & 0.769446 & 0.666830 & 0.668572 \\
\hline 2.071193 & 1.071193 & $1.744754^{*}$ & 40 & 1.076203 & 0.813546 & 0.816561 \\
\hline 1.798158 & 1.053542 & $1.070833^{*}$ & 41 & 0.818620 & 0.818620 & 0.817634 \\
\hline 1.780719 & 1.034832 & $1.118034^{*}$ & 42 & 1.130693 & 0.863011 & 0.813182 \\
\hline 1.412480 & 0.912480 & $0.886346^{*}$ & 43 & 0.832936 & 0.785317 & 0.784932 \\
\hline 1.798158 & 1.173049 & $1.322876^{*}$ & 44 & 0.873277 & 0.873277 & 0.871473 \\
\hline 1.105822 & 0.829249 & 0.779283 & 45 & 0.844820 & 0.844820 & 0.844864 \\
\hline 1.817557 & 1.000000 & 0.926535 & 46 & 0.973114 & 0.882205 & 0.881655 \\
\hline 1.108042 & 0.899812 & 0.788900 & 47 & 0.744386 & 0.787865 & 0.790079 \\
\hline 1.412480 & 0.912480 & $0.961267^{*}$ & 48 & 1.096688 & 0.870037 & 0.876180 \\
\hline 0.976421 & 0.885771 & 0.878823 & 49 & 0.744132 & 0.696513 & 0.697158 \\
\hline 1.735502 & 1.000000 & $0.906786^{*}$ & 50 & 0.821890 & 0.832182 & 0.762408 \\
\hline 0.892444 & 0.741007 & 0.737610 & 51 & 0.817323 & 0.770458 & 0.771552 \\
\hline 1.105822 & 0.829249 & 0.897528 & 52 & 0.884117 & 0.837232 & 0.837318 \\
\hline 1.097307 & 0.769689 & 0.760264 & 53 & 0.829958 & 0.791497 & 0.725979 \\
\hline 1.253606 & 0.771116 & 0.773805 & 54 & 0.881762 & 0.789677 & 0.794864 \\
\hline 1.001057 & 0.876057 & 0.873548 & 55 & 0.795133 & 0.795133 & 0.798504 \\
\hline 1.108042 & 0.899812 & 0.824180 & 56 & 0.981635 & 0.828677 & 0.833854 \\
\hline 1.001556 & 0.911763 & 0.911536 & 57 & 0.834201 & 0.834201 & 0.833807 \\
\hline 1.276501 & 0.776501 & 0.800391 & 58 & 0.793283 & 0.744371 & 0.745533 \\
\hline 1.130693 & 0.863011 & 0.805421 & 59 & 0.710444 & 0.724352 & 0.710444 \\
\hline 0.976421 & 0.885771 & 0.879312 & 60 & 1.056320 & 0.749787 & 0.748270 \\
\hline 0.973114 & 0.882205 & 0.880877 & 61 & 0.719386 & 0.715829 & 0.717979 \\
\hline 1.703144 & 1.000000 & 0.744935 & 62 & 0.771883 & 0.838550 & 0.845276 \\
\hline 0.821890 & 0.832182 & 0.764098 & 63 & 0.847851 & 0.736979 & 0.750188 \\
\hline 0.892444 & 0.744403 & 0.744403 & 64 & 0.842522 & 0.781042 & 0.779800 \\
\hline 0.881762 & 0.789677 & 0.795451 & 65 & 0.753091 & 0.753091 & 0.753171 \\
\hline 1.039662 & 0.764535 & 0.775919 & 66 & 0.797466 & 0.779343 & 0.788725 \\
\hline 0.793283 & 0.744371 & 0.745590 & 67 & 0.708427 & 0.738730 & 0.731833 \\
\hline 1.097307 & 0.769689 & 0.760264 & 68 & 0.803940 & 0.803940 & 0.805077 \\
\hline 0.771883 & 0.838550 & 0.845276 & 69 & 0.740573 & 0.732708 & 0.733806 \\
\hline 1.015064 & 0.866076 & 0.860459 & 70 & 0.865028 & 0.782493 & 0.779932 \\
\hline 0.797466 & 0.779343 & 0.788725 & 71 & 0.750488 & 0.750488 & 0.750488 \\
\hline 1.001057 & 0.876057 & 0.874371 & 72 & 1.062100 & 0.754145 & 0.765297 \\
\hline 0.865028 & 0.782493 & 0.779932 & 74 & 0.867916 & 0.816491 & 0.815422 \\
\hline 1.163674 & 0.830341 & 0.829482 & 75 & 0.729046 & 0.684061 & 0.685155 \\
\hline 0.867916 & 0.816491 & 0.815422 & 76 & 0.771532 & 0.768761 & 0.702507 \\
\hline 1.001556 & 0.911763 & 0.911624 & 77 & 0.690677 & 0.690677 & 0.690677 \\
\hline
\end{tabular}


TABLE 2 (continued)

\begin{tabular}{|c|c|c|c|c|c|c|c|}
\hline$\underline{k}$ & $\theta$ & $\theta \#$ & $\psi$ & $\underline{k}$ & $\theta$ & $\theta \#$ & $\psi$ \\
\hline 78 & 0.769446 & 666830 & 572 & 130 & 3091 & 3091 & 0.753171 \\
\hline 80 & 0.806030 & .774123 & 0.773105 & 132 & 0.834519 & 762402 & 0.763278 \\
\hline 81 & 0.757028 & 757028 & 0.757028 & 134 & 0.708427 & .738730 & .731833 \\
\hline 82 & 0.818620 & 818620 & 0.817634 & 138 & 0.740573 & 732708 & .73380 \\
\hline 84 & 0.811175 & 0.805972 & 0.805906 & 140 & 0.737551 & .764606 & 0.769112 \\
\hline 85 & 0.694146 & 0.694146 & 0.694146 & 142 & 0.750488 & .750488 & 0.750488 \\
\hline 86 & 0.832936 & 0.785317 & 0.784932 & 143 & 0.713437 & .713437 & 0.713437 \\
\hline 87 & 0.688240 & 0.759668 & 0.688240 & 144 & 0.753220 & 680366 & 0.680366 \\
\hline 88 & 0.780273 & 0.742456 & 0.741766 & 150 & 0.729046 & 684061 & .685155 \\
\hline 90 & 0.844820 & 0.844820 & 0.844864 & 154 & 0.690677 & 90677 & 690677 \\
\hline 91 & 0.688707 & 0.688707 & 0.688 & 156 & 0.789471 & 789471 & .790208 \\
\hline 92 & 0.728679 & 0.720354 & 0.719 & 162 & 0.757028 & 7028 & 0.757028 \\
\hline 93 & 0.691390 & 0.758056 & 0.691390 & 163 & 0.719154 & 0.719154 & 0.719154 \\
\hline 94 & 0.744386 & 0.787865 & 0.790048 & 168 & 0.6998 & 579 & 0.674416 \\
\hline 95 & 0.698739 & 732484 & 0.698 & 169 & 0.718525 & 1525 & 0.71852 \\
\hline 96 & 0.825985 & 11 & 0.742 & 170 & 0.694146 & 4146 & 0.694146 \\
\hline 98 & 0.744132 & 0.696513 & 0.697303 & 174 & 0.688240 & 0.759668 & 0.688240 \\
\hline 99 & 0.740587 & 0.691390 & 0.691390 & 180 & 0.723937 & .723937 & 0.723937 \\
\hline 100 & 0.813770 & 0.813770 & 0.812489 & 182 & 0.688707 & 8707 & 0.688707 \\
\hline 102 & 0.817323 & 0.770458 & 0.771552 & 186 & 0.691390 & 758056 & 0.691390 \\
\hline 104 & 0.723333 & 0.767924 & 0.765581 & 190 & 0.698739 & 2484 & 0.698739 \\
\hline 105 & 0.653897 & 0.653897 & 0.653897 & 198 & 0.740587 & 0.691390 & 0.691390 \\
\hline 106 & 0.829958 & 0.791497 & 0.725979 & 210 & 0.653897 & 0.653897 & 0.653897 \\
\hline 108 & 0.764890 & 0.722346 & 0.661992 & 216 & & 698739 & 0.698739 \\
\hline 110 & 0.7 & & & 222 & & 294 & $69873 !$ \\
\hline 111 & 0.698739 & 0.754294 & 0.698 & 234 & 0.698 & 8739 & 0.69873 \\
\hline 112 & 0.740472 & 0.740472 & 0.742195 & 242 & 0.711 & 617 & 0.71143 \\
\hline 114 & 0.834201 & 0.834201 & 0.834201 & 243 & 0.719154 & 0.731499 & 0.71915 \\
\hline 116 & 0.764606 & 0.751262 & 0.700136 & 250 & 0.709028 & 0.709827 & 0.70902 \\
\hline 117 & 0.698739 & 0.698739 & 0.698739 & 256 & 0.714815 & 0.714815 & 0.71481 \\
\hline 118 & 0.710444 & 0.724352 & 0.710444 & 286 & 0.713437 & 0.713437 & 0.71343 \\
\hline 120 & 1.032367 & 0.672746 & 0.672746 & 326 & 0.719154 & 0.719154 & 0.719154 \\
\hline 121 & 0.711433 & 0.717617 & 0.711433 & 338 & 0.718525 & 0.718525 & 0.718525 \\
\hline 122 & 0.719386 & 0.715829 & 0.716087 & 360 & 0.707926 & 0.707926 & 0.707926 \\
\hline 124 & & & & 420 & & 0.688445 & 0.688445 \\
\hline 125 & 0.709028 & 0.709827 & 0.709028 & 432 & 0.717112 & 0.717112 & 0.71711 \\
\hline 126 & 0.847851 & 0.736979 & 0.750188 & 486 & 0.719154 & 0.731499 & 0.71915 \\
\hline
\end{tabular}

$\left(^{*}\right)$ The $\psi$-bounds for small $k$ and $x$ are distorted by powers of $p=2,3$. If the range $0-10^{10}$ is replaced by $1500-10^{10}$, the bounds are much improved, as shown below. Alternatively, a $\psi$-bound of 1.0 holds for $X_{0} \leq x \leq 10^{10}$, with $X_{0}$ as below:

\begin{tabular}{|c|c|}
\hline$k$ & $\psi: 1500-10^{10}$ \\
\hline 1 & 0.790594 \\
\hline 2 & 0.805589 \\
\hline 3 & 0.764104 \\
\hline 4 & 0.879669 \\
\hline 5 & 0.806086 \\
\hline 6 & 0.785064 \\
\hline 10 & 0.792735 \\
\hline 12 & 0.862547 \\
\hline
\end{tabular}


Table 2 was computed by using a sieve. First, $\theta$ and $\psi$ were computed for each arithmetic progression. All three functions

$$
\begin{gathered}
{[\theta(x, k, l)-x / \varphi(k)] / \sqrt{x}, \quad[\psi(x, k, l)-x / \varphi(k)] / \sqrt{x},} \\
{[\theta(x ; k, l)-(x-N(k, l) \sqrt{x}) / \varphi(k)] / \sqrt{x}}
\end{gathered}
$$

are monotone decreasing between their jumps at primes or prime powers. Thus, to determine their maximal absolute values over a given interval, it suffices to check their values at the endpoints of the interval, and their left and right limits at primes and prime powers within the interval.

Considerable roundoff error can accumulate in summing $10^{9}$ or more floating point numbers. To assure the accuracy of the computations, upper and lower bounds for $\theta$ and $\psi$ were computed by first rounding all the logarithms up and down to the nearest $2^{-30}$. After this truncation, the upper and lower bounds could essentially be computed by integer arithmetic, e.g. without roundoff error, and $\theta$ and $\psi$ were replaced by their bounds in such a way as to assure that Table 2 gave rigorous upper bounds. (This choice of the truncation would have allowed the computation to continue as high as $1.7 \times 10^{10}$, considerably beyond its actual limit of $10^{10}$.) Finally, the values were rounded up in their last digit.

\section{ACKNOWLEDGEMENTS}

Ramaré wishes first of all to thank his advisor J.-M. Deshouillers who, after having suggested this problem, gave good directions for the research. Then we warmly thank L. Reboul who verified many of the computations, and Professors M. Balazard and A. Odlyzko for their useful comments. We also wish to thank the referee, who found some errors in an earlier version of the paper.

\section{REFERENCES}

1. E. Landau, Handbuch der Lehre von der Verteilung der Primzahlen, with an appendix by P. Bateman, 3rd edition, Chelsea, New York, 1974.

2. J. van de Lune, H. J. J. te Riele, and D. T. Winter, On the zeros of the Riemann zeta function in the critical strip. IV, Math. Comp. 46 (1986), 667-681.

3. K. S. McCurley, Explicit estimates for functions of primes in arithmetic progressions, Ph.D. thesis, University of Illinois at Urbana-Champagne, 1981.

4. _ Explicit zero-free regions for Dirichlet L-functions, J. Number Theory 19 (1984), $7-32$.

5. __ Explicit estimates for the error term in the prime number theorem for arithmetic progressions, Math. Comp. 42 (1984), 265-285.

6. _ـ Explicit estimates for $\theta(X ; 3, l)$ and $\psi(X ; 3, l)$, Math. Comp. 42 (1984), 287-296.

7. W. Press, B. Flannery, S. Teukolsky, and W. Vetterling, Numerical recipes, Cambridge Univ. Press, Cambridge, 1986.

8. J. B. Rosser, Explicit bounds for some functions of prime numbers, Amer. J. Math. 63 (1941), 211-232.

9. J. B. Rosser and L. Schoenfeld, Sharper bounds for the Chebyshev functions $\theta(X)$ and $\psi(X)$, Math. Comp. 29 (1975), 243-269.

10. R. Rumely, Numerical computations concerning the ERH, Math. Comp. 62 (1993), 415-440.

11. L. Schoenfeld, Sharper bounds for the Chebyshev functions $\theta(X)$ and $\psi(X)$. II, Math. Comp. 30 (1976), 337-360. 
12. S. B. Stechkin, Rational inequalities and zeros of the Riemann zeta-function, Trudy Mat. Inst. Steklov. 189 (1989), 110-116; English transl. in Proc. Steklov Inst. Math. 189 (1990), $127-134$.

13. R. Terras, A Miller algorithm for an incomplete Bessel function, J. Comput. Phys. 39 (1981), $233-240$.

Département de Mathématiques, Université de Nancy I, URA 750, 54506 Vandoeuvre Cedex, France

Department of Mathematics, University of Georgia, Athens, Georgia 30602 